Review Article

\title{
Clinical Interventions for Hyperacusis in Adults: A Scoping Review to Assess the Current Position and Determine Priorities for Research
}

\author{
Kathryn Fackrell, ${ }^{1,2}$ Iskra Potgieter, ${ }^{1}$ Giriraj S. Shekhawat, ${ }^{3,4}$ David M. Baguley, ${ }^{1,2}$ \\ Magdalena Sereda, ${ }^{1,2}$ and Derek J. Hoare ${ }^{1,2}$ \\ ${ }^{1}$ NIHR Nottingham Biomedical Research Centre, Ropewalk House, Nottingham, UK \\ ${ }^{2}$ Otology and Hearing Group, Division of Clinical Neuroscience, School of Medicine, University of Nottingham, Nottingham, UK \\ ${ }^{3}$ Health Systems and Audiology, The University of Auckland, Auckland, New Zealand \\ ${ }^{4}$ Tinnitus Research Initiative, Regensburg, Germany
}

Correspondence should be addressed to Kathryn Fackrell; kathryn.fackrell@nottingham.ac.uk

Received 31 May 2017; Accepted 16 August 2017; Published 9 October 2017

Academic Editor: Alberto Raggi

Copyright ( $) 2017$ Kathryn Fackrell et al. This is an open access article distributed under the Creative Commons Attribution License, which permits unrestricted use, distribution, and reproduction in any medium, provided the original work is properly cited.

\begin{abstract}
Background. There is no universally accepted definition for hyperacusis, but in general it is characterised by decreased sound tolerance to ordinary environmental sounds. Despite hyperacusis being prevalent and having significant clinical implications, much remains unknown about current management strategies. Purpose. To establish the current position of research on hyperacusis and identify research gaps to direct future research. Design and Sample. Using an established methodological framework, electronic and manual searches of databases and journals identified 43 records that met our inclusion criteria. Incorporating content and thematic analysis approaches, the definitions of hyperacusis, management strategies, and outcome measures were catalogued. Results. Only $67 \%$ of the studies provided a definition of hyperacusis, such as "reduced tolerance" or "oversensitivity to sound." Assessments and outcome measures included Loudness Discomfort Levels, the Hyperacusis Questionnaire, and Tinnitus Retraining Therapy (TRT) interview. Management strategies reported were Cognitive Behavioural Therapy, TRT, devices, pharmacological therapy, and surgery. Conclusions. Management strategies were typically evaluated in patients reporting hyperacusis as a secondary complaint or as part of a symptom set. As such the outcomes reported only provided an indication of their effectiveness for hyperacusis. Randomised Controlled Trials are needed to evaluate the effectiveness of management strategies for patients experiencing hyperacusis.
\end{abstract}

\section{Introduction}

Hyperacusis is the perception of everyday environmental sound as being overwhelmingly loud or intense. Other terminology in use includes reduced, decreased, or collapsed sound tolerance. It differs from phonophobia which is an episodic sound intolerance experienced by some people during migraine attacks, sometimes associated with other sensory sensitivities, and which abates as the attack recedes [1]. It can also be differentiated from misophonia which is an acquired aversive reaction to specific human generated sounds such as eating sound or breathing, the response being characterised by anger and sometimes rage $[2,3]$.
As with other subjective symptoms, data on the prevalence of hyperacusis is strongly influenced by how the enquiry about the symptom was worded. In children and adolescents, the variability in wording of the enquiry in prevalence studies has been found to be so great as to render comparison across studies to be meaningless [4]. In a study of 7096 11-yearold children in the UK, 3.7\% answered affirmatively to the question "do you ever experience oversensitivity or distress to particular sounds?" [5]. In adults, variability of the questions asked across studies of hyperacusis also significantly impacts on the ability to identify prevalence figures. Using the question "Do you consider yourself sensitive to everyday sounds?," Andersson and colleagues [6] found that of 1,174 
adults that answered the question via either the Internet or post, $8.6 \%$ (95\% CI $=7.0-10.0)$ responded affirmatively. Another Swedish study [7] in adults asked 3406 participants, "Do you have a hard time tolerating everyday sounds that you believe most other people can tolerate?" and found 9.2\% responded affirmatively, with $1.9 \%$ reporting that they had been diagnosed with hyperacusis by a physician.

There is an apparent association between hyperacusis and tinnitus, with $86 \%$ of adult patients with a primary complaint of hyperacusis experience tinnitus [8] and $40 \%$ of patients with a primary complaint of tinnitus experience hyperacusis as well [9]. Many people with troublesome hyperacusis have normal or age appropriate hearing thresholds, but cochlear hearing loss has also been reported [10]. Medical conditions associated with hyperacusis include closed head injury, depression, posttraumatic stress syndrome, Williams syndrome, and pain syndromes such as fibromyalgia [1]. The physiological mechanisms that underpin hyperacusis are not well understood, and there is no compelling animal model. A consensus is emerging in the auditory neuroscience literature that hyperacusis may be associated with a sustained and persistent increase in central auditory gain [11].

A framework with which to categorise patients with hyperacusis has been proposed [12]. The subtypes consisted of hyperacusis that is characterised by loudness, annoyance, fear, or pain. This schema was not based upon empirical data and may serve to illustrate the various characteristics that can define the lived experience of hyperacusis, rather than to support diagnosis or treatment. Regardless, hyperacusis is a complex symptom which can have negative effects on daily functioning such as hearing, sleep, concentration, and emotional well-being that can vary daily and between individuals. Management, therefore, can be complex with approaches taken including cognitive behaviour therapy, Tinnitus Retraining Therapy, or sound therapy [13]. Complex interventions such as these need to be developed and evaluated in a systematic way to have confidence in the effectiveness of the intervention for the given population [14].

The purpose of this scoping review is to identify research gaps in existing literature and elements that should underpin the design of any new studies. The aim of this scoping review is to consider the current position of research on hyperacusis in adults. Specifically, the objective here is to identify

(i) how hyperacusis is currently defined in research studies,

(ii) How it is currently measured (i.e., what measures are used for diagnosis and outcome and are they adequate?),

(iii) What the level of evidence is for current management options (i.e., what has been previously studied and to what extent?).

\section{Materials and Methods}

This review is reported according to the methodological framework developed by Arksey and O'Malley [15] using the six-stage process. In this process (1) the purpose and research questions were defined, (2) relevant studies were identified, (3) studies were selected using an iterative approach through title, abstract, and full-text screening by three members of team (KF, IP, and GSS), (4) data were extracted and charted by two members of the team (KF, IP), (5) the results were collated, summarised, and reported, and (6) two clinical experts, who were not involved in the data extraction or collating and summarising results, were consulted and reviewed the findings.

2.1. Eligibility Criteria. To be included, records were required to report studies in which adults ( $\geq 18$ years old) reported hyperacusis as a primary complaint or secondary complaint or as part of a symptom set. Records were included where management strategies (i.e., interventions) were tested to address hyperacusis. Records were eligible from Randomised Controlled Trials (RCTs), nonrandomised control trials, cohort studies, case series, and case studies. Review articles including systematic reviews, epidemiology articles, and any sources reporting personal/expert opinions were excluded. No records were excluded based on controls used, outcomes reached, timing, setting, or study design. Records were excluded for studies reporting misophonia, phonophobia, and loudness recruitment.

All included records were published in the English language. Where multiple eligible unique records pertaining to a single trial were identified, the record that was published first was included and any secondary analyses of the data were excluded.

2.2. Search Strategy. The search strategy followed a recommended three-step approach $[16,17]$. In Step 1, to test keywords and search terms, a limited search in PsycINFO and Embase was conducted, checking the availability of relevant titles and abstracts. This allowed us to develop search term combinations to use across a wider search in step two. The search strategy included hyperacusis and terms for identifying research studies, such as intervention, therapy, treatment, management, assessment, outcome, and diagnoses (diagnostic) with narrow terms such as measure and test (Table 1). In Step 2, to identify relevant research studies, electronic databases of peer reviewed journals were searched in the Cochrane Ear, Nose and Throat Disorders Group Trials Register; the Cochrane Central Register of Controlled Trials (CENTRAL); PubMed; Embase; PsycINFO; Scopus; Cumulative Index to Nursing and Allied Health Literature (CINAHL); Web of Science; the International Standard Randomised Controlled Trial Number (ISRCTN) registry; ClinicalTrials.gov; the International Clinical Trials Registry Platform (ICTRP); and Google Scholar. Specific search term strategies were applied in each search engine, searching article topics, titles, abstracts, and keywords. Where possible, filters were applied to retrieve articles in the English language and using human participants only. There was no restriction in the search period as we wanted to include all available research up until the present time.

In Step 3, to seek further eligible documents for inclusion, we performed manual searches of the reference lists of any relevant review articles which had hyperacusis in the title. 
TABLE 1: Search term strategies for hyperacusis interventions and outcome measures. CENTRAL = Cochrane Central Register of Controlled Trials; CINAHL = Cumulative Index to Nursing and Allied Health Literature; ISRCTN = International Standard Randomised Controlled Trial Number; ICTRP = the International Clinical Trials Registry Platform.

\begin{tabular}{|c|c|}
\hline Search terms & Search engine \\
\hline $\begin{array}{l}\text { Hyperacus }{ }^{*} \text { AND } \text { assess }^{*} \text { OR measure* } \text { OR test }^{*} \\
\text { OR outcom* OR Diagnos* OR defin* }{ }^{*} \text { OR treat }{ }^{*} \text { OR } \\
\text { manag* OR therap* OR interv }^{*}\end{array}$ & Embase, PsycINFO, CENTRAL, Scopus, Web of Science \\
\hline $\begin{array}{l}\text { Hyperacus }^{*} \text { AND }\left[\left(\text { assess }^{*} \text { OR measure* OR test* }\right.\right. \\
\text { OR diagnos* OR defin OR outcom* OR therap* OR } \\
\text { interv }^{*} \text { OR treat }\end{array}$ & Web of Science, Scopus, PubMed, CENTRAL, CINAHL Plus \\
\hline Hyperacus ${ }^{*}$ & Cochrane ENT Disorders Group Trials Register \\
\hline Hyperacusis & ClinicalTrials.gov, ISRCTN, ICTRP \\
\hline $\begin{array}{l}\text { Hyperacusis AND [assessment OR measurement OR } \\
\text { test OR Outcome OR diagnosis OR definition OR } \\
\text { treatment OR Therapy OR intervention] }\end{array}$ & Google Scholar \\
\hline
\end{tabular}

In addition, manual searches of the most common journals (determined using the interquartile rule for outliers) in which eligible records had been sourced were conducted. The final manual search was conducted in April 2017.

2.3. Study Selection. Articles identified through electronic and manual searches were exported with citations, title, and abstract into Endnote where duplicates were removed. Search records were screened independently by two researchers out of a team of three (KF, IP, and GSS), first screening by title and abstract and then by full text. When disagreements regarding the inclusion or exclusion of any given record arose, the two researchers discussed their rationale until agreement was reached or a third researcher $(\mathrm{DH})$ was consulted to reach a majority decision.

2.4. Data Extraction. A data extraction form was developed and piloted on two included records and was subsequently modified following team discussions. Data from each article were extracted by two researchers (KF and IP). Data were extracted on study characteristics, definition of hyperacusis, assessment measures used, and interventions (Box 1).

\section{Results}

3.1. Study Selection. Figure 1 illustrates the flow of study records identified, screened, included, and excluded (with reasons for exclusion). Electronic searches yielded an initial set of 1708 records. Duplicates were removed and of the remaining 792 records, 710 were excluded because the title and abstract indicated that the article did not fit our eligibility criteria. Most commonly the studies excluded did not focus on hyperacusis or did not report treatment of hyperacusis. Manual searches of included records in review articles identified a further 27 potential articles which were subjected to full-text screening. Manual searches in the selected journals identified one additional eligible record. Sixty-seven records were excluded at the full-text screening stage. Commonly, this was because the record did not report on the treatment or management of hyperacusis or was based on expert opinion or a review of the literature. For 1 record the reference was incomplete and could not be found. Full-text records could not be located for a further 2 records. None of these records could be traced, regardless of support from the University of Nottingham librarian. The electronic and manual searches created a final list of 43 eligible full-text records for data collection.

3.2. Study Characteristics. Of the 43 included full-text articles, there were 34 journal articles, six conference papers, and three book chapters. Articles were published from 1984, with the majority published after 2001 and the most recent being published in 2016. Records were predominantly reporting studies from the USA, UK, Australia, India, South Korea, and European countries. Of the included records, 23 were case studies [19, 25-27, 34-38, 40-43, 49-58], seven were cohort studies $[28,29,39,44,46,59,60]$, five were RCTs $[18,20,21,30,47]$, four were Nonrandomised Controlled Trials $[22,23,31,48]$, and four were retrospective studies $[24,32,33,45]$.

3.3. Population Characteristics. Of the 43 included records, 16 studies [18, 19, 21-25, 38, 39, 41-43, 55-57, 60] included patients reporting hyperacusis as a primary complaint. Of these studies, nine (53\%) were case studies and only two (12\%) were RCTs (Table 2). Equally, 16 studies [34-37, 40, 44, 45, $47,49-54,58,59]$ included patients reporting hyperacusis as part of a set of symptoms, the most common of which were tinnitus, hearing loss, vertigo, or aural fullness. The remaining 11 studies included patients reporting hyperacusis as a secondary complaint to tinnitus [20, 26-28, 30-33, 48] and/or hearing loss [29, 46]. Duration of the participants hyperacusis was not reported in 29 studies [20-26, 28-33, 38, $39,42-48,50-53,56,57,59]$. For the remaining 14 studies $[18,19,27,34-37,40,41,49,54,55,58,60]$, the duration of hyperacusis reported ranged from 6 weeks in a surgical case study [55] to 27 years in an "acoustic training" case study [40].

3.4. Current Definitions for Hyperacusis. Fourteen studies [26-28, 31-33, 44, 45, 47, 48, 51, 52, 54, 57] did not provide a working definition of hyperacusis. Across the remaining 
First author surname

Year of publication

Study Title

Publication type: journal article, report, conference paper, undergraduate dissertation, postgraduate dissertation,

book chapter, webpage, or other (please specify)

Country where study was conducted

Aim of study

Terminology used to describe hyperacusis

Study design: case report, case series, cohort study, retrospective study, non-randomised control trial, randomised control trial, or other

Study design (if other)

Methods: questionnaire, focus groups, interviews, audiometric tests, clinical tests, medical/physical investigations, or combination

Sample size for primary analysis (not follow up)

Study population: patients, general public, students or combination

Setting

Age

Gender

Hyperacusis complaint: primary, secondary or as part of symptom set

What are the comorbid conditions/syndrome/symptom set?

Hyperacusis-related symptoms reported

Average duration of hyperacusis in years (SD)

Measures used for screening of hyperacusis to the study

Assessment time points

Outcome measures used on patients with hyperacusis

Treatment primarily aimed at hyperacusis

Nature of therapeutic interventions reported (summarise key components)

Treatment outcomes from questionnaires

Treatment outcomes from LDLs

Treatment outcomes - anecdotal reports

Study limitations

Further research priorities specified

Box 1: Data extraction fields.

studies, common terminologies were used to define hyperacusis, with four main themes identified (Figure 2).

One theme that emerged from the definitions focused on "reductions" in sound tolerance [24, 34, 38, 43, 50, 60]. Authors described hyperacusis as "a reduction of normal tolerance for everyday sounds" [38], "decreased sound tolerance" [24], "lowered threshold for sound tolerance" [50], or "the collapse of loudness tolerance" [43]. In contrast to this, other studies emphasised the "increased sensitivity" to sound [20, 29, 35, 39, 40, 42, 46, 53, 55]. This theme included descriptors such as "hypersensitivity," "oversensitivity," or "abnormal sensitivity" to define the degree of tolerance to sounds (Figure 2). One author used descriptors that indicated increased loudness, "a disproportionate growth in subjective loudness of sounds" [49]. Other definitions referred to "intolerance" to sounds that had been deemed as ordinary [18, $19,21,30,37,41,58,59]$. Authors referred to "noise intolerance to ordinary sounds" [58], "unusual intolerance" $[18,19]$, or "intolerance to the loudness of sounds that most individuals deem to be tolerable" [21]. The last theme to emerge included definitions that referred to the physical or emotional reaction to the sound, such as "subject exhibits negative reactions" [25], "abnormal, generally painful perception of loudness" [23], or the "response or reaction from the auditory cortex" $[22,36]$.

Underlying these themes, throughout, was the common concept of the tolerance to sound being different from "normal." Definitions referred to sound tolerance of "normal listeners" [38], “a normal person" [40], “a typical person” [19], or "others" [43] in comparison to sound tolerance of patients with hyperacusis. For example, Formby et al. [30] described hyperacusis as a "general intolerance to the loudness of sounds that would not typically be bothersome for most individuals." In some cases, the response to "ordinary" or "everyday" environmental sounds was described as "unusual" $[18,19,55]$ or "abnormal" $[22,23,36,42]$, and in one case the response was described as "exaggerated or inappropriate" [19]. 


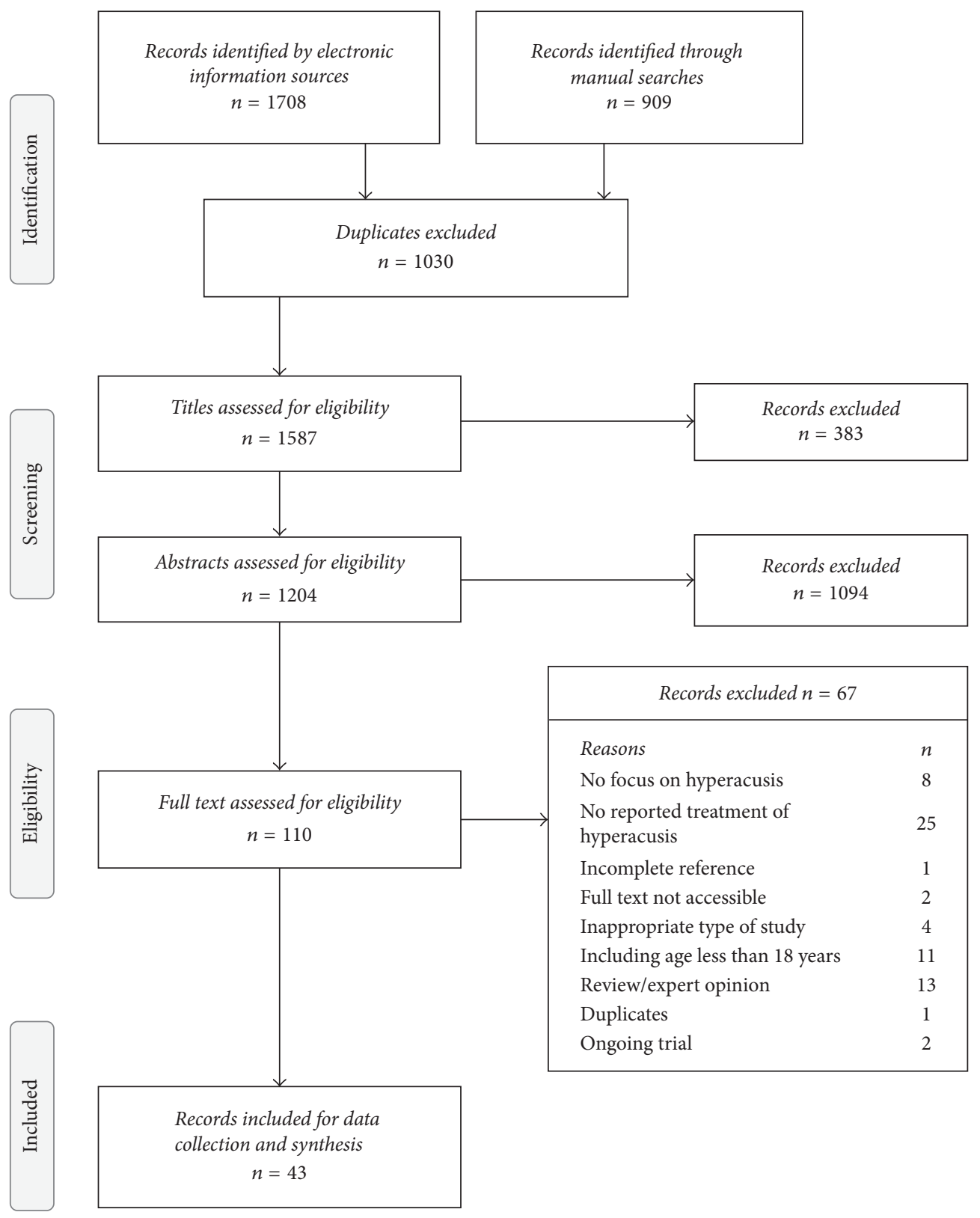

FIGURE 1: Flow chart of stages of study selection process.

Across the themes reductions, sensitivity, and intolerance, some authors noted within their working definitions the overt physical and emotional responses evoked by the perception of sounds. These authors highlighted the "bothersome" [30, 39], "distressing" [20], or "disabling" [37] nature of the sounds, the "discomfort" $[19,40,43,49,55]$ or "pain" [23, 49] caused by the perception of sound, or the negative reaction to exposure to sound [25]. In one case, Silverstein et al. [58] listed associated symptoms of "emotional, social and physical" reactions to hyperacusis within their working definition.

3.5. Assessment Tools Used for Diagnosis and Outcome. In six studies (14\%), the assessment tools used to quantify or diagnosis hyperacusis were not stated [20, 21, 39, 43, 47, 59].
Sixteen studies used clinical interviews (history and examination) to assess patient symptoms [28, 29, 35, 36, 41, 42, 46, 4957]. Most of these studies were case series and reports, with some implying that interviews were used but not explicitly stating this.

Of the remaining 21 studies [18, 19, 22-27, 30-34, 37, $38,40,44,45,48,58,60]$, audiometric measures (Loudness Discomfort Levels (LDLs), Maximum Comfort Levels (MCLs), and Dynamic Range (DR)) and patient selfreported measurement tools (Baltimore questionnaire (VAS) [61], Sound Hypersensitivity Questionnaire [62], Hyperacusis Questionnaire (HQ) [63], Hyperacusis test (12-item) [38], a non-validated Hyperacusis Questionnaire adapted from Geraüschüberempfindlichkeit (GÜF [64]) [60], and Tinnitus 


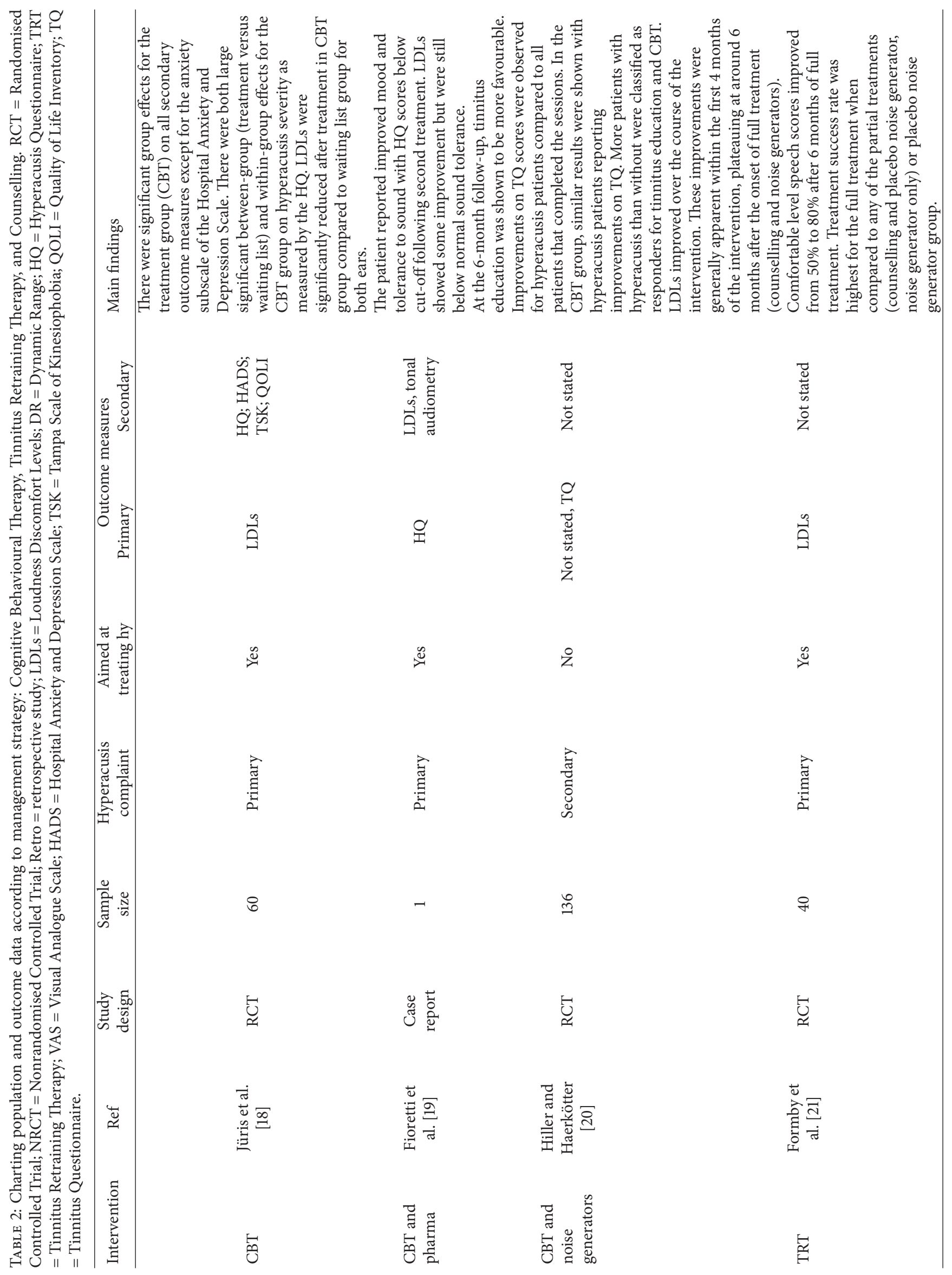




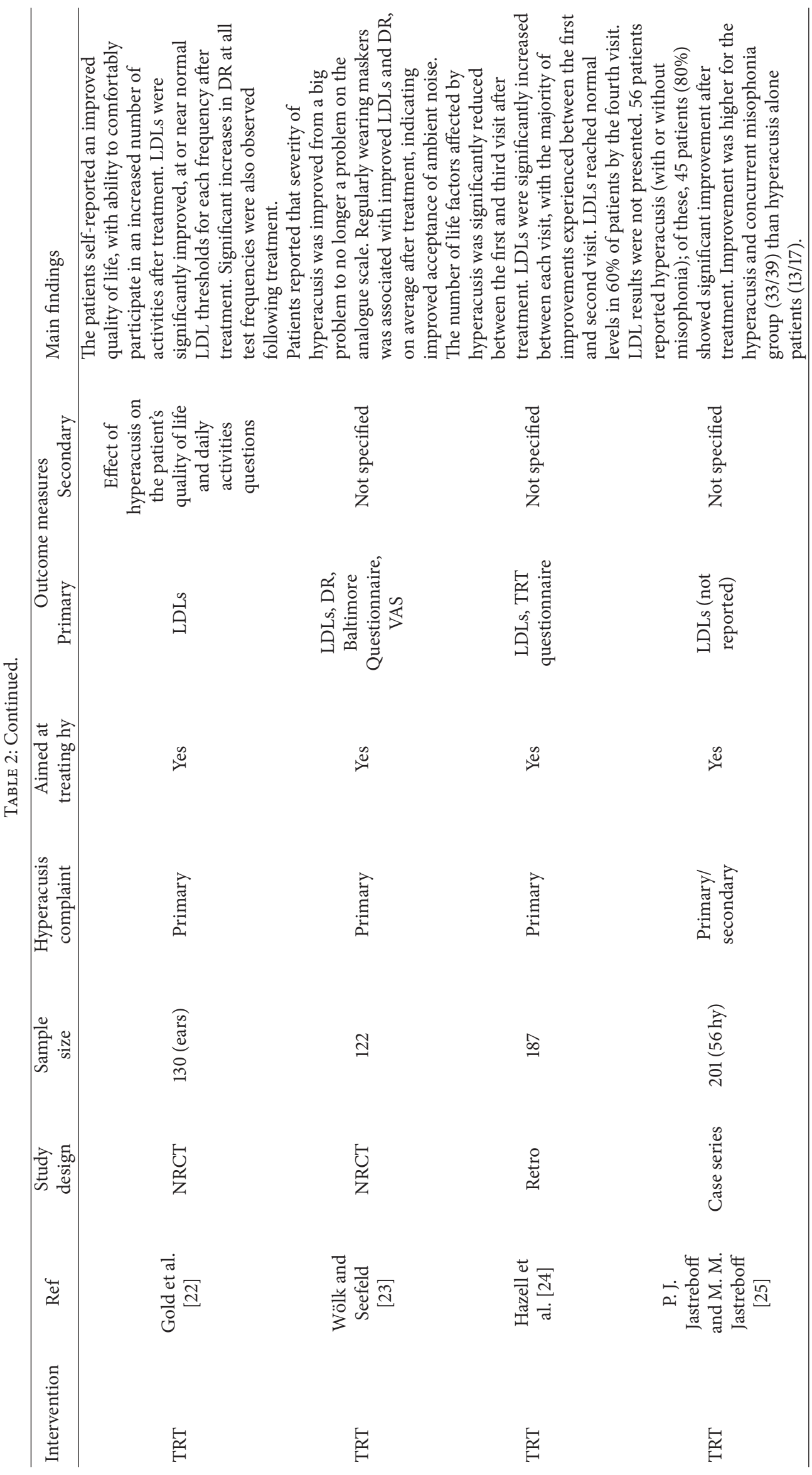




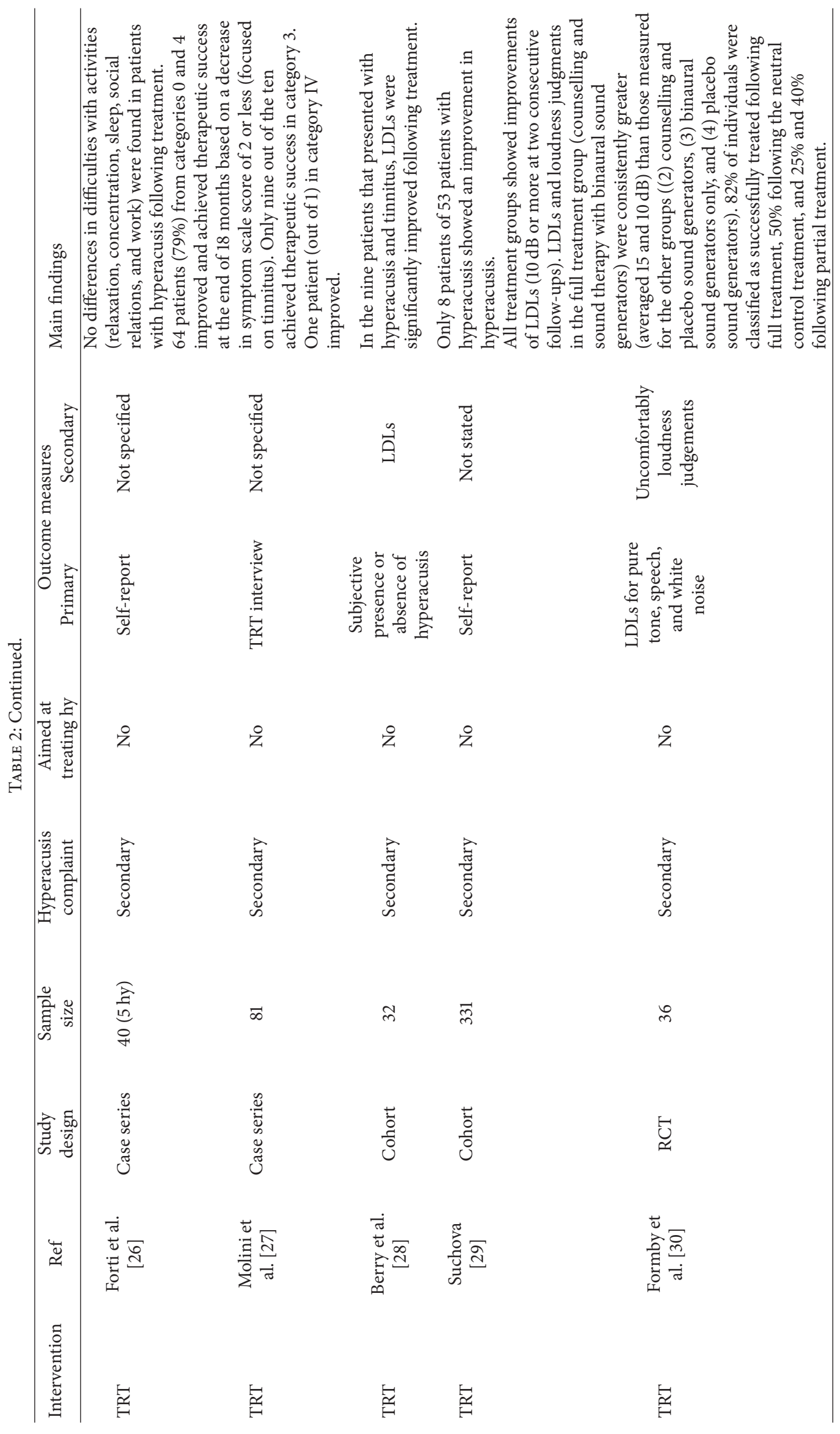




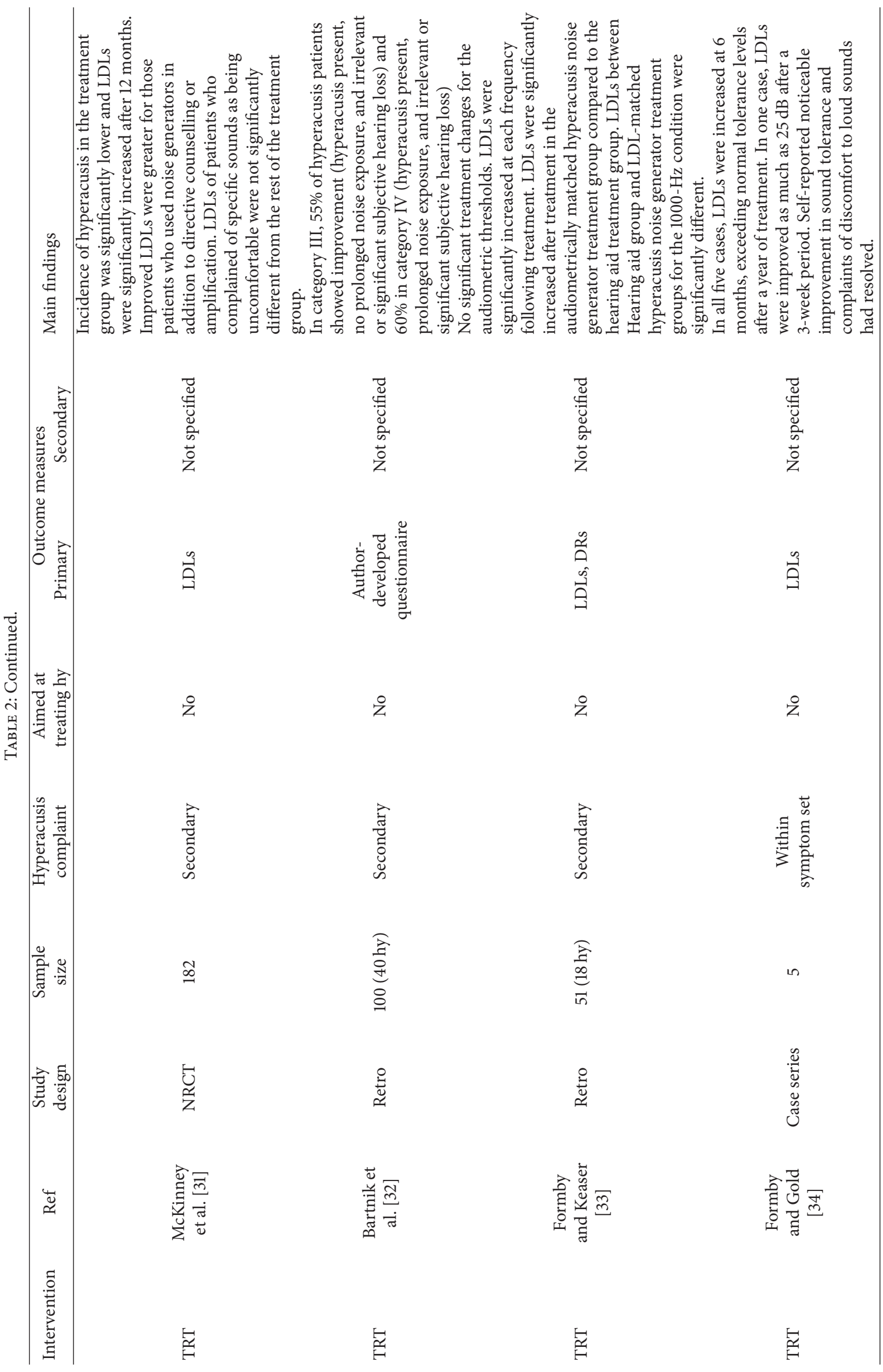




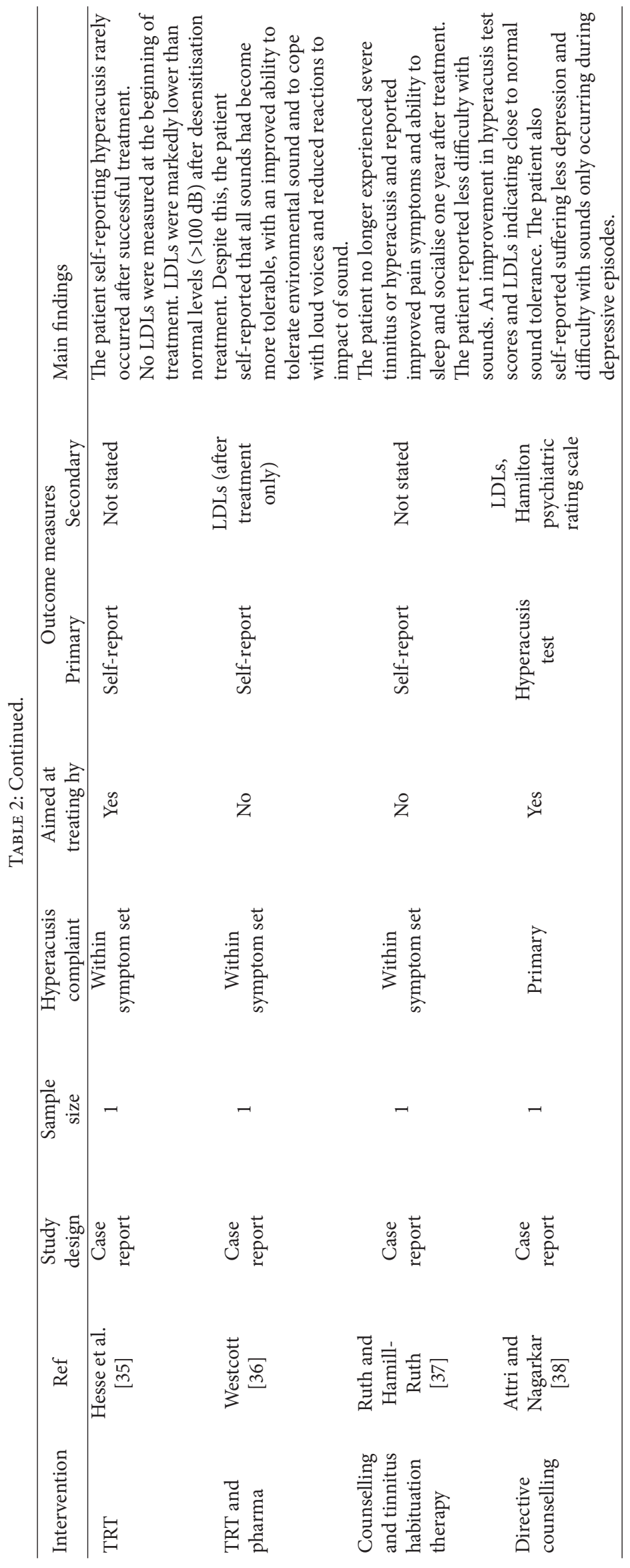




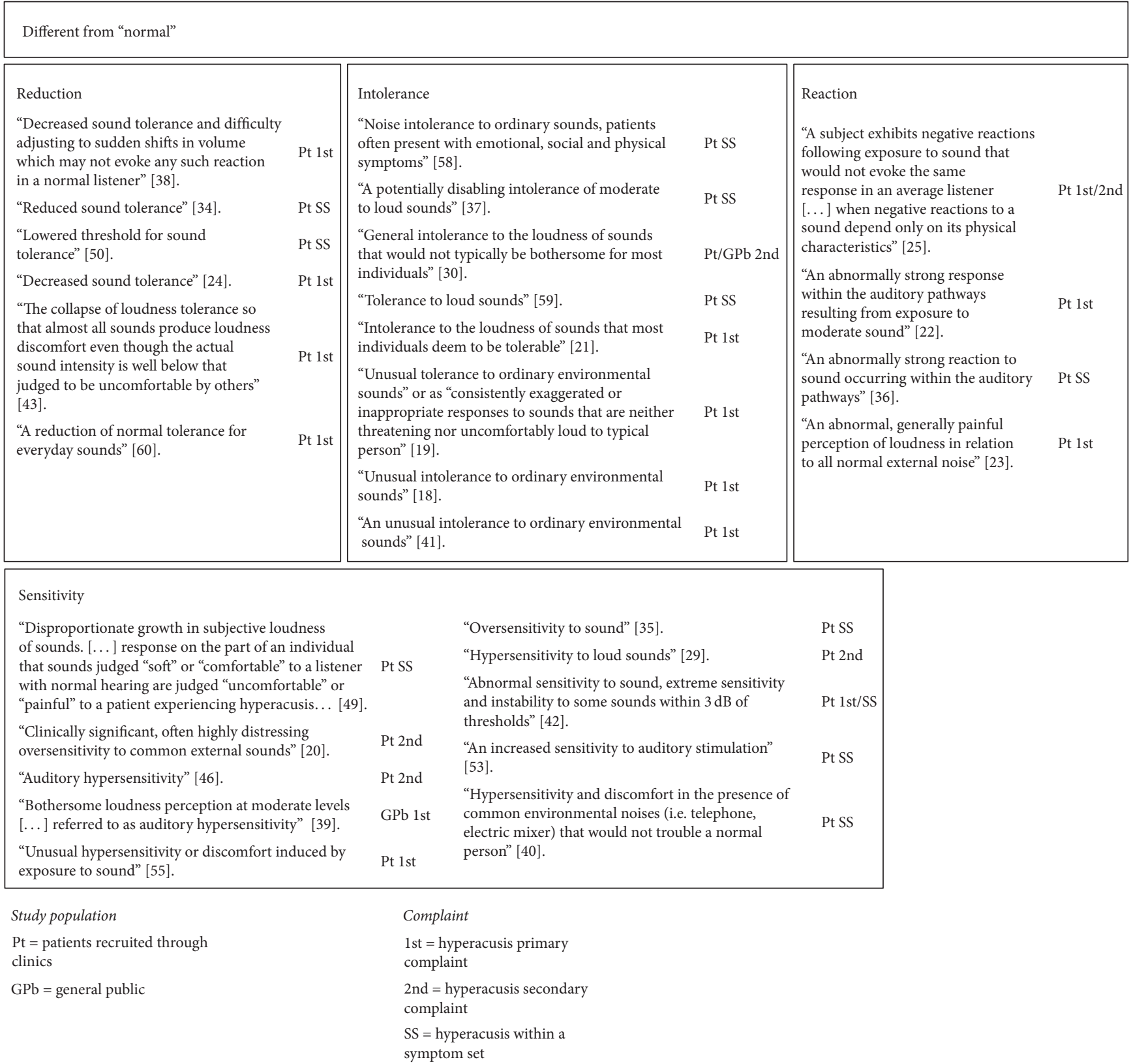

FIGURE 2: The four themes and underlying theme to emerge from the content of the working definitions of hyperacusis reported in the included records.

Retraining Therapy (TRT) assessment interview [65]) were reported to assess hyperacusis. Most commonly reported were LDLs, the HQ and TRT assessment interview. The six studies $[18,22,24,30,31,37]$ that reported using LDLs varied in the $\mathrm{dB}$ values used to quantify hyperacusis. Gold et al. [22] and Hazell et al. [24] specified that average LDLs should be below $100 \mathrm{~dB} \mathrm{HL}$ for $1,2,3$, and $8 \mathrm{kHz}$ in both ears (pure tones), whilst Jüris et al. [18] and Formby et al. [30] specified that LDLs using pure tones should be $\leq 90 \mathrm{~dB}$ HL in at least one ear at the frequencies of $0.5,1$, and $2 \mathrm{kHz}$ or from at least two frequencies in the range $0.5 \mathrm{kHz}$ to $4 \mathrm{kHz}$, respectively. In contrast, McKinney et al. [31] specified less commonly used LDLs using pure tones of less than 88.39 dB SPL and
81.24 dB SPL to classify individuals as having hyperacusis with normal hearing and hearing loss, respectively. Ruth and Hamill-Ruth [37] did not specify any criteria for LDLs. A similar case was found across the four studies reporting the HQ [19, 45, 48, 58]. Only one study [19] specifically identified a value on the HQ that would diagnose hyperacusis (36 points). Seven studies used the TRT assessment interview to classify patients into one of five categories associated with tinnitus and hyperacusis [24-27, 32-34]. Of these studies, only Hazell et al. [24] explicitly referred to categories 3 and 4 (hyperacusis present).

The most commonly specified posttreatment outcome measures were LDLs and the HQ (Tables 2-4). Nineteen 


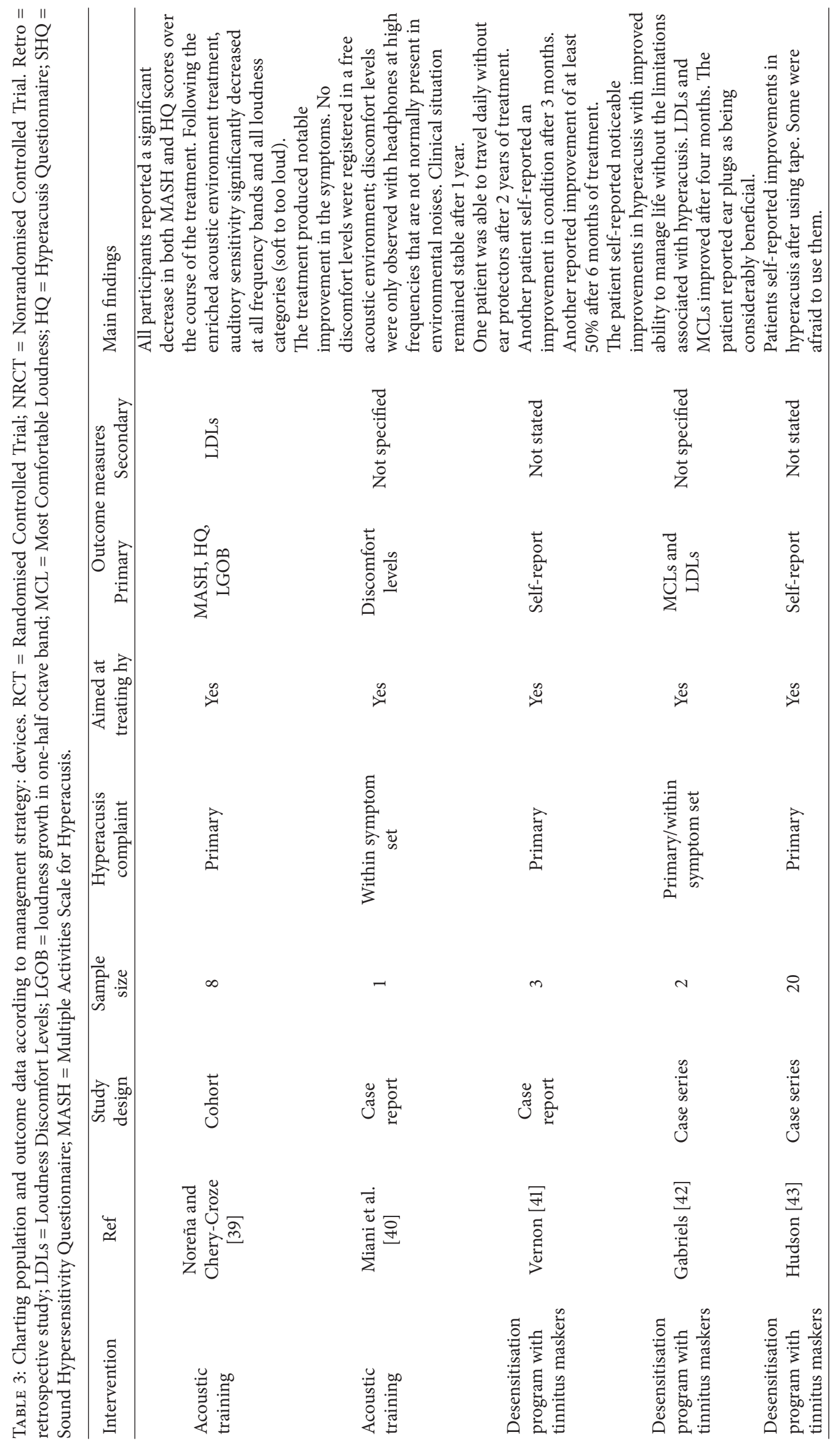




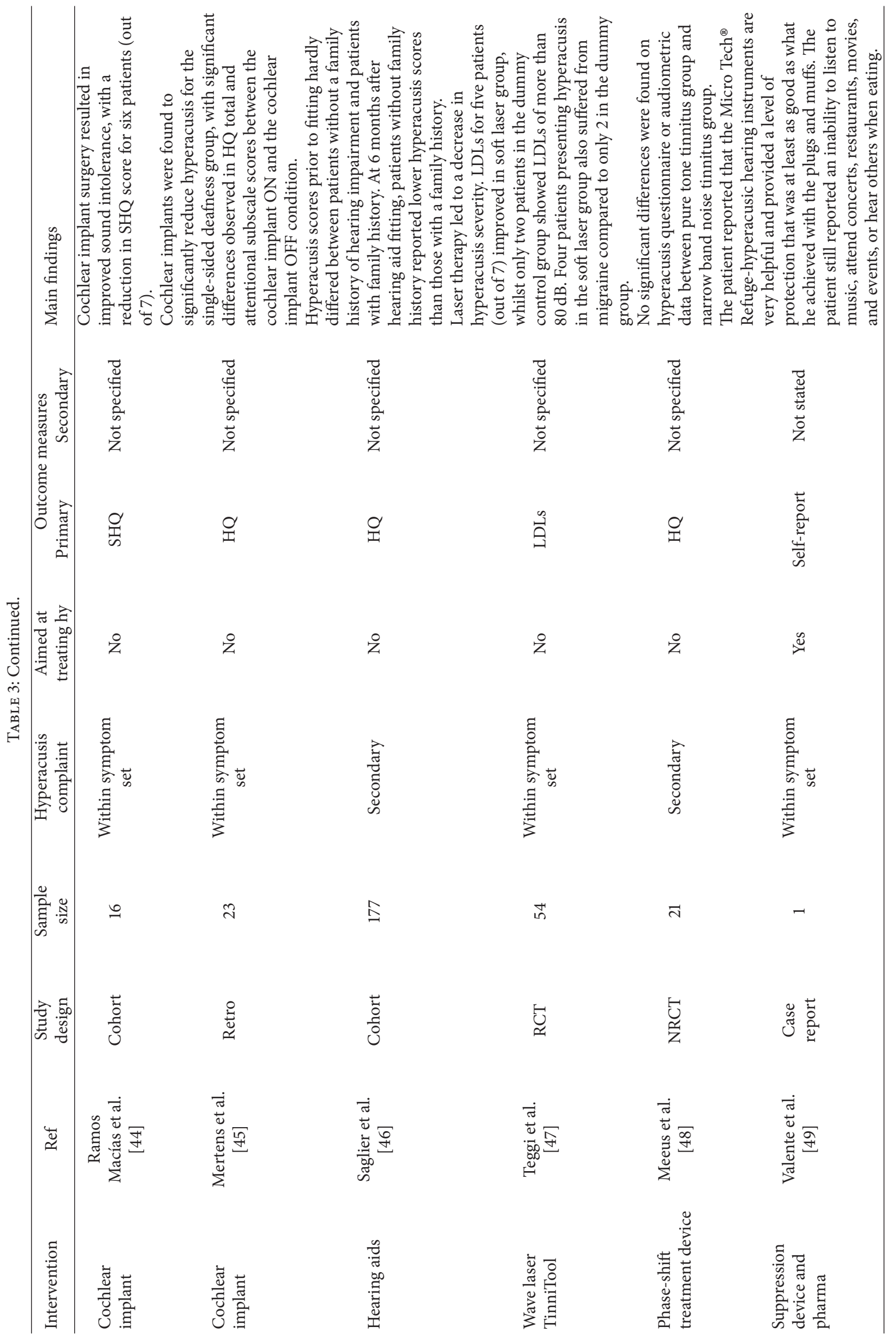



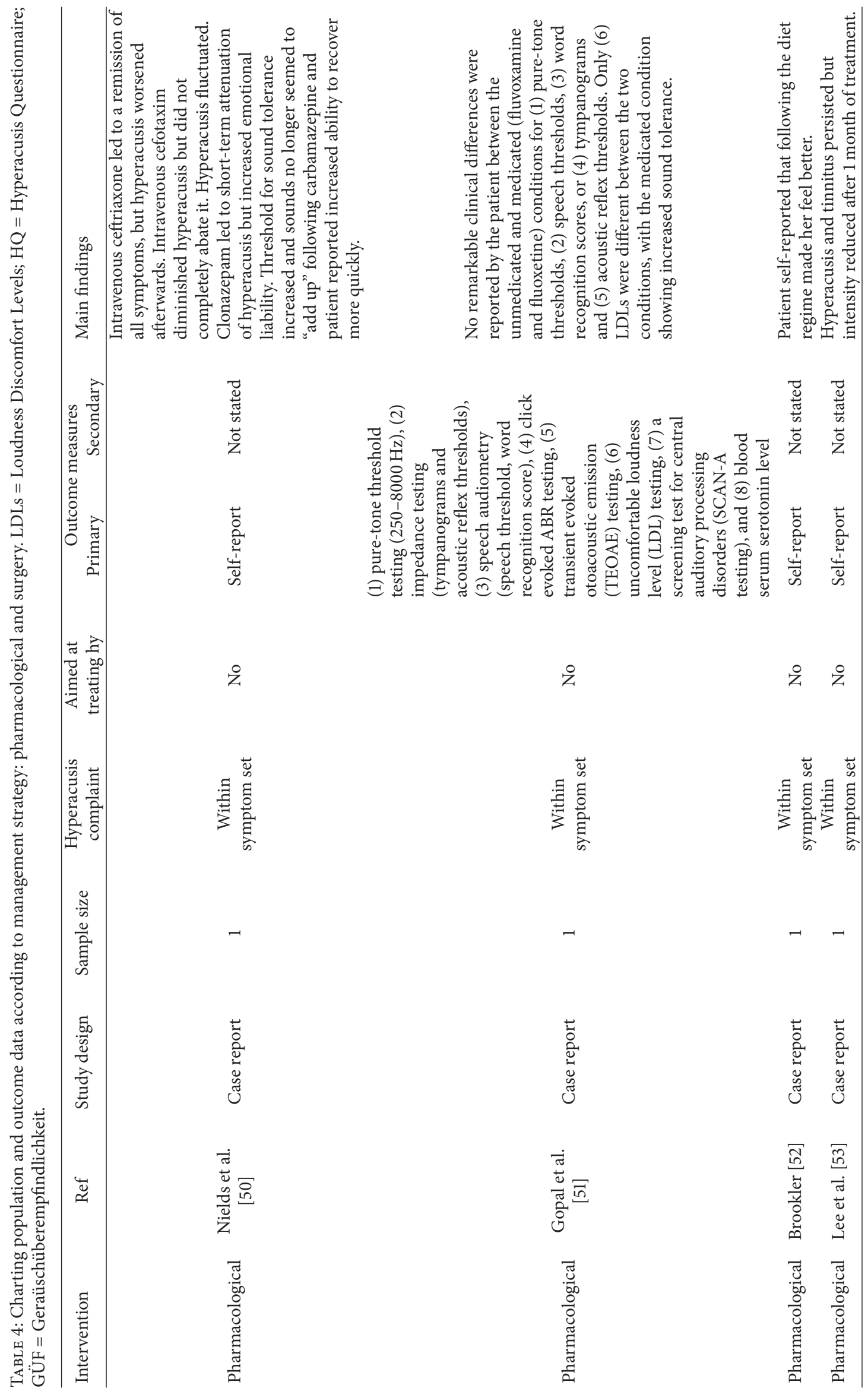

そ)

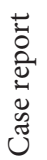

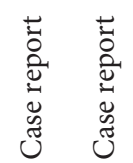

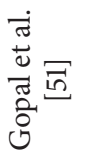

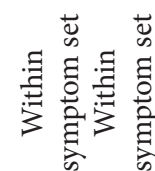

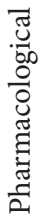

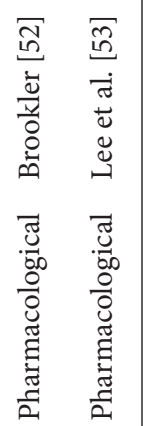




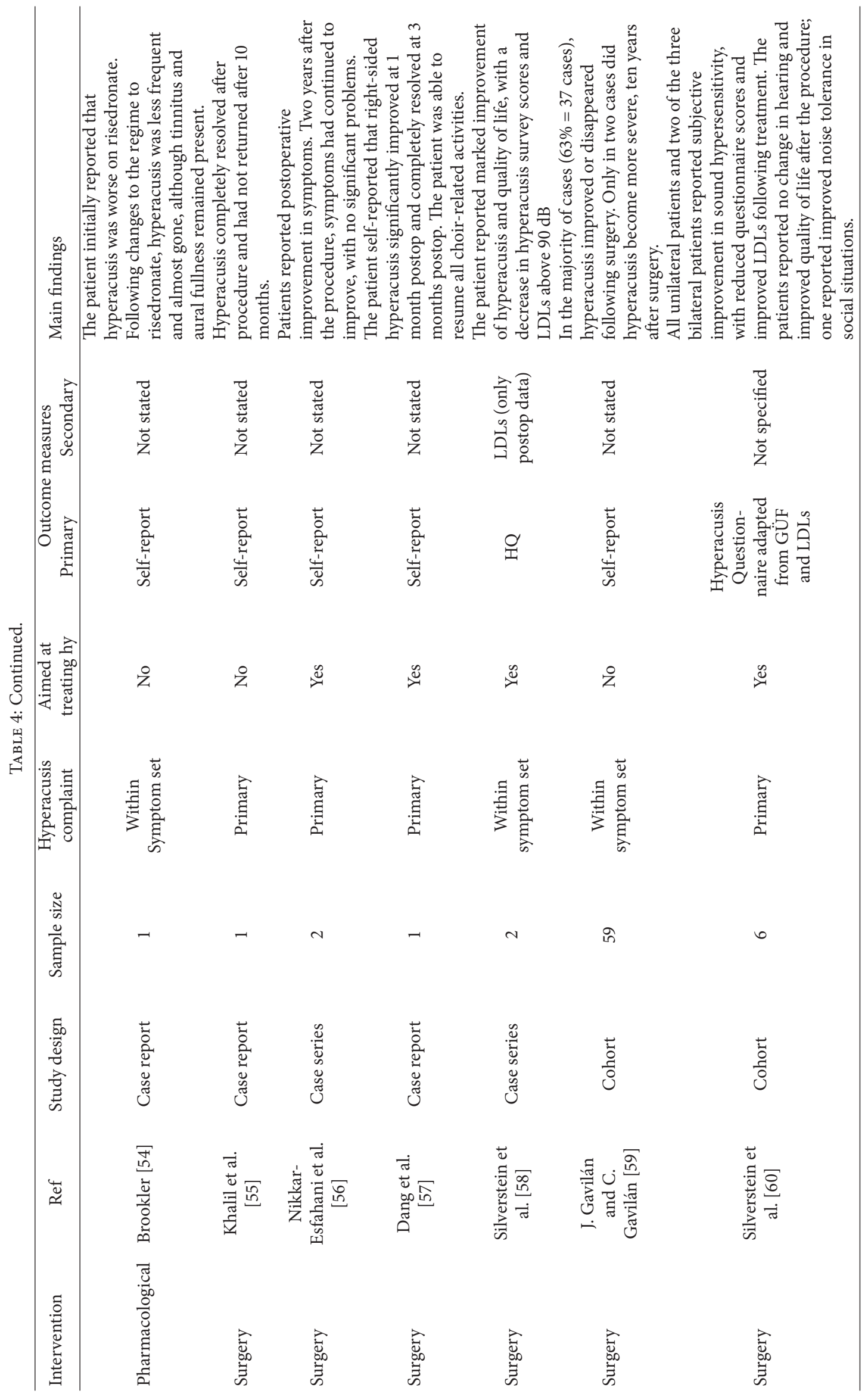


studies reported increases in LDLs after treatment as either a primary $[18,21-25,30,31,33,34,42,47,60]$ or secondary outcome $[19,28,36,38,39,58]$. Six studies reported changes in HQ scores as the primary outcome $[19,39,45,46,48$, 58] or secondary outcome measure [18]. Of the 43 included records, 13 case studies [26, 35-37, 41, 43, 49, 50, 52-57] and two cohort studies $[29,59]$ relied on patient self-reported change in hyperacusis through clinical assessment at followup. One study [20] did not state a hyperacusis-specific outcome measure, only reporting the Tinnitus Questionnaire [66] as an outcome measure. The remaining studies reported a variety of different outcome measures, ranging from single item visual analogue scales to multi-item hyperacusis-specific questionnaires such as Multiple Activity Scale for Hyperacusis (MASH) [67] and Sound Hypersensitivity Questionnaire [62].

There is reasonable body of evidence on the development and reliability of the HQ as a diagnostic tool $[63,68]$, but the validity and reliability of the HQ as an outcome measure are yet to be fully examined. Questions have been raised on the appropriateness of the items in the questionnaire and the need of validation in a population with a primary complaint of hyperacusis is known [68]. Evidence for the reliability of LDLs is variable $[69,70]$ with reliability depending on a number of factors including instructions given to patients [71] and choice of sound stimuli [8]. Importantly, there are inconsistencies in the relationship between LDLs and selfreport sound tolerance, with LDLs (using pure tones or speech sounds) often failing to reliably relate to self-reports of tolerance sounds in daily life [72].

3.6. Current Management Options for Hyperacusis. All 43 included records reported potential treatment benefits for hyperacusis (Tables 2-4). However, only nineteen studies (44\%) sought to evaluate interventions or management strategies specifically aimed at reducing hyperacusis $[18,19$, $21-25,35,38-43,49,56-58,60]$. For the most part, the studies explored interventions that were primarily aimed at reducing tinnitus. Management strategies explored were Cognitive Behavioural Therapy (CBT), TRT, counselling, devices, pharmacological therapy, and surgery.

3.7. Cognitive Behavioural Therapy. Three studies explored the potential benefits of CBT [18-20] (Table 2). Jüris et al. [18] reported an RCT investigating the benefits of CBT for patients experiencing hyperacusis. Treatment comprised general CBT principles that were aimed to educate, target overt emotional reactions to sounds though graded exposure to sounds, reduce stress though relaxation, and provide patients with the tools to manage more difficult situations and restart activities (behavioural activation). After treatment, patients in the CBT group showed a significant reduction in hyperacusis severity as assessed by the HQ and an increase in LDLs from baseline, compared to the waiting list group. Only small effects were observed for quality of life and depression, and symptoms of anxiety were unchanged. Fioretti et al. [19] reported a case study in which a patient with hyperacusis underwent a four-month course of pharmacological therapy with bilateral sound generators (graded sound exposure) and
CBT to target fear of sounds. Reduced hyperacusis-related symptoms were reported. Following this, the patient suspended treatment and underwent chemotherapy for breast cancer. Eight months later, after cancer treatment, the patient reported worsening of hyperacusis symptoms. Serotonin reuptake inhibitors and a further 4 months of CBT were prescribed. Following treatment, the patient reported an improvement in mood (depression, hostility, and sadness) and sound tolerance. In this case, no details were provided on the components of CBT. Hiller and Haerkötter [20] reported an RCT investigating CBT and the possible additional effects of sound stimulation on improving severity of tinnitus. In this study, one group received treatment comprised of an educational component (tinnitus education) in which patients learned about and applied psychological concepts such as "vicious cycle" and "coping cycle" to their personal situations with tinnitus. The second group completed ten sessions of CBT, including education (avoiding silence), changing thought processes (relaxation), diverting attention, and identifying avoidant behaviours and short/long terms consequences of behaviour. Half of each group were supplied with sound stimulation through behind-the-ear broadband white noise generators for each ear, with volume controls for graded increases. Following treatment, greater improvements in tinnitus severity were reported for patients with hyperacusis compared to those without hyperacusis. Notably, hyperacusis was not measured after treatment in this study.

3.8. Tinnitus Retraining Therapy. Sixteen studies explored the use of TRT for patients with hyperacusis as a primary complaint [21-25] or a secondary complaint [26-33] or as part of a symptom set [34-36] (Table 2.).

Nine studies [24-29, 32, 34, 35] focused on a classic TRT protocol to elevate hyperacusis with/without tinnitus. These studies incorporated a component of counselling including educational training in which the Jastreboff neurophysiological model is described to explain treatment and demystify the patients' experience. Guidance is given about avoidance behaviour (e.g., use of earplugs, avoiding environment sounds, or avoiding quiet) and the application of desensitising sound and sound enrichment was discussed. The depth of counselling and sound components depends on the treatment category ( $0-4$ : presence of tinnitus, hearing loss, hyperacusis, or noise exposure) assigned; for categories 1-2, sound generators are recommended; for categories 3-4 aimed at hyperacusis, bilateral open-fitting sound generators are fitted with instructions to gradually increase the sound daily to be tolerable without difficulties. Sound enrichment techniques are also used, in which digitally produced nature sounds are slowly reintroduced [24-29, 32, 34, 35]. Eight of these studies reported improvements in hyperacusis [25, $27,29,32,35]$, three of which reported increased LDLs $[24,28,34]$ following treatment. Hazell et al. [24] reported that, after 2 years of treatment, LDLs were well within the normal range $(>100 \mathrm{~dB})$ in over $60 \%$ of patients. In a case series, Formby and Gold [34] found that individual patients were reporting "noticeable subjective improvement in sound tolerance," resolution of complaints of discomfort, and in 
one case "complete resolve of sound tolerance problems." In a case report, Hesse et al. [35] described the need for the patient to build up a good therapeutic relationship before starting the sound exposure and generator component of therapy. This led to hyperacusis only occurring on very rare occasions. Both Suchova [29] and Molini et al. [27] reported that only a small number of patients with hyperacusis showed improvement following TRT. Molini et al. [27] reported that only one patient in hyperacusis category 3 achieved therapeutic success (a decrease of 2 or less on the scale of symptoms, a Tinnitus Handicap Inventory Grade 1, and an awareness of tinnitus value of less than or equal to $10 \%$ of the patient's wakefulness). In contrast, Forti et al. [26] found that TRT led to patients reporting no differences in difficulties with activities (relaxation, concentration, sleep, social relations, and work) following the treatment.

Three studies evaluated the effectiveness of using sound generators alongside directive counselling with patients reporting a primary complaint of hyperacusis [21], tinnitus [31], or hearing loss [30]. All three studies reported that LDLs had significantly improved over the course of treatment. In an RCT, Formby et al. [21] showed a clear pattern of LDLs initially increasing and then plateauing at 6 months after the onset of full treatment (counselling and noise generators). All three studies also reported greater treatment success and improvements of hyperacusis in patients who used noise generators in addition to directive counselling than those using part of the treatments (counselling alone, counselling and placebo noise generator, or noise generator only). In another RCT, Formby et al. [30] found that changes in judgements of uncomfortable loudness for the full treatment group after treatment averaged $15 \mathrm{~dB}-10 \mathrm{~dB}$, compared to the changes of $5 \mathrm{~dB}$ or less in the other groups.

Gold et al. [22] investigated functional auditory changes demonstrated by increases in LDLs and DR during TRT for patients with tinnitus and hyperacusis. Following treatment, both LDLs and DR increased from the initial assessment whilst hearing threshold did not change significantly. The authors concluded that the DR can be increased following TRT. Patients self-reported an increase in quality of life and a decrease in the number of daily activities affected by tolerance problems. Similarly, Wölk and Seefeld [23] reported that regular use of maskers (set at hearing threshold and slowly increased) improved LDLs and DR and reduced severity of hyperacusis to "no longer a problem." Formby and Keaser [33] explored the sound therapy component of TRT and potential treatment benefits for tinnitus patients using hearing aids (HA) versus audiometrically matched or LDL-matched tinnitus patients with hyperacusis using noise generators. Increases in LDLs and DR at follow-up were observed for both the audiometrically matched and LDLmatched groups. In the HA versus audiometrically matched noise generator condition, changes in hearing thresholds between groups were nominal, but patients using noise generators showed significant increments in LDLs compared to the HA group. In the HA versus LDL-matched noise generator condition, the changes in LDLs at $1 \mathrm{kHz}$ were greater for the LDL-matched group than the HA group; differences were negligible at other frequencies.
In a case study of a patient with posttraumatic stress disorder reporting hyperacusis as a symptom, Westcott [36] reported that, following an initial treatment of antidepressants (a selective serotonin uptake inhibitor/a serotonin and noradrenaline reuptake inhibitor) for sound intolerance attributed to anxiety and depression, a TRT desensitisation program was carried out to help increase tolerance to environmental sounds. The patient self-reported an improved ability to cope with loud sounds, reduced reaction to unexpected loud sounds, and the ability to actively pursue her own piano playing.

One case report followed some of the TRT principles without explicitly stating this method [37]. Ruth and HamillRuth [37] applied counselling with tinnitus habituation therapy (bilateral fitted in-the-ear-noise generators) to improve daily activities, relaxation, and mood monitoring in a patient experiencing both tinnitus and hyperacusis. In addition to therapy, the patient was prescribed Baclofen for pain and sleep disturbance. After one year, the patient no longer experienced tinnitus or hyperacusis and reported an improved ability to sleep and socialise.

3.9. Counselling Alone. Attri and Nagarkar [38] reported a case study using hyperacusis-focused directive counselling to educate the patient on the auditory system and mechanisms of hyperacusis (Table 2). Guidance and advice were given on avoiding silence, overprotection of ears, and use of background sounds to desensitise hyperacusis. The patient reported a reduction in HQ scores from 23 points (moderate) to 15 points (close to normal) and improved tolerance to sounds, with difficulties tending to occur only during depressive episodes.

3.10. Devices Alone. Eleven studies reported the effects of using different devices on patients with hyperacusis as a primary complaint or secondary complaint or as part of a symptom set (Table 3). Two studies [39, 40] reported "acoustic training" for hyperacusis which notably improved symptoms in the long term, with symptoms remaining in remission over a year later. These studies slightly differed in methodology. The seven "acoustic training" sessions (administered every 5 days) reported by Miani et al. [40] consisted of six to ten stimulations in which a narrow band noise was sent to an acoustic free field, before a $60 \mathrm{~dB}$ HL pure tone (other types of sounds were also used in later sessions) was sent through headphones for about 3 minutes followed by a pause of 3 minutes. The intensity of the sound stimulus and duration were increased in $5 \mathrm{~dB}$ HL steps (up to a total increase of $35 \mathrm{~dB} \mathrm{HL}$ ) and up to 5 minutes of exposure, until the last session, when the sound in the headphones reached $95 \mathrm{~dB}$ HL in all frequencies. High frequencies used in the sessions led to reports of discomfort by patients when listening via headphones, but not in the acoustic free environment. These sounds were not reflective of normal environmental sounds, so authors were not concerned. In contrast, Noreña and Chery-Croze [39] used passive exposure to an enriched acoustic environment (EAE). Sounds used were pure tones (based on the cut-off frequency of hearing loss) presented in random order for the duration of $100 \mathrm{~ms}$ with pauses 
between of $100 \mathrm{~ms}$. Participants listened to the EAE through headphones for 1-3 hours a day at a just audible level. Auditory sensitivity significantly decreased at all frequency bands, from 2 weeks onwards. The impact of hyperacusis on activities and daily functioning was reduced, with all participants reporting significant decrease in both $\mathrm{MASH}$ and HQ scores over the course of the treatment.

Three studies reported desensitisation programs using broadband tinnitus maskers [41, 42] and pink noise tape cassettes [43]. To desensitise patients, sounds were presented in each ear through wearable tinnitus maskers or through headphones (for pink noise) and started at a threshold the patient could tolerate and gradually increased in loudness over the sessions. In the case series reported by Vernon [41], the patients wore ear plugs during the day and carried out the desensitisation program at night. All three studies reported improvements in hyperacusis, although some patients did not comply with the use of the tape cassettes as they were afraid to use them because they believed that it would aggravate their hyperacusis. One patient reported improved ability to manage life without hyperacusis limitations [42].

One RCT [47] evaluated the effectiveness of a continuous wave laser TinniTool versus a dummy laser device. A laser probe was inserted into a specifically designed headset which projects the laser beam (power output $5 \mathrm{~mW}$ ) onto the tympanic membrane though a 17-degree diverging lens (creating a spot size of $1 \mathrm{~cm}$ ) for 20 minutes a day, resulting in an energy density of around $6 \mathrm{~J}$ at the membrane. The placebo dummy device, apart from activation of the laser beam, reproduced all aspects of laser therapy. Tinnitus patients in the laser therapy group reported decreased hyperacusis. Seven patients in the laser therapy group and eight patients in the placebo group reported LDLs lower than $80 \mathrm{~dB}$ at baseline; of these, LDLs improved in five patients in laser therapy group compared to two patients in placebo group following treatment.

Two studies $[44,45]$ reported the long term effects of cochlear implants (CI) treatment in patients with unilateral hearing loss and tinnitus. Mertens et al. [45] found that hyperacusis only significantly reduced for the single-sided deafness group and not the asymmetric hearing loss group. Significant differences were observed in HQ total and the attentional subscale scores between the CI-ON and the CIOFF condition for the single-sided deafness group. Ramos Macías et al. [44] reported that CI use resulted in improved sound tolerance for six out of seven patients, who showed a reduction in Sound Hypersensitivity Questionnaire scores [62]. Only one patient showed decreased sound tolerance problems. Saglier et al. [46] investigated the impact of family history of hearing impairment on rehabilitation using HAs and reported that hyperacusis scores were more improved in patients without a family history following fitting of HAs than those with a family history.

One study [48] reported that 21 sessions using a tinnitus progressive phase-shift treatment device ("Phase-outTM"), which presented a sound that resembles the frequency and amplitude of patients' own tinnitus but shifts $6^{\circ}$ every 30 seconds, did not significantly change scores on the HQ or audiometric data between a pure tone tinnitus group and a narrow band noise tinnitus group. All measurements remained unchanged after therapy.

One case study [49] reported that diazide and methylprednisolone improved unilateral hearing loss but did not diminish hyperacusis. Following fitting of a custommade binaural full-concha, unvented in-the-ear compression device (Micro Tech ${ }^{\circledR}$ Refuge-hyperacusic), the patient reported that the devices were very helpful and provided a level of protection that was at least as good as what he achieved with the plugs and muffs, but reported that he still could not carry out social activities (listening to music, attending concerts, restaurants, movies, and events, or hearing others when eating).

3.11. Pharmacological Therapy. Pharmacological therapy was reported in five case studies [50-54] where hyperacusis was part of a set of symptoms (Table 4). One case study [52] reports a patient placed on a diet to address metabolic factors, bisphosphonate and calcium, for otosclerosis. The patient reported feeling better until the treatment regime was discontinued, when symptoms of dizziness and hyperacusis returned. The patient was prescribed bisphosphonate risedronate and reported reduced complaints of symptoms and feeling best when the diet regime was followed. In another case study, Brookler [54] reported a patient prescribed metformin, risedronate (30 mg twice weekly), calcium, vitamin $\mathrm{D}$, and sodium monofluorophosphate who reported worsening of hyperacusis on days when they took risedronate. Alternating the regime with etidronate $(400 \mathrm{mg} /$ day for 2 weeks) and risedronate ( $30 \mathrm{mg}$ twice weekly for 4 weeks) did not improve hyperacusis but increasing risedronate intake to $30 \mathrm{mg}$ twice weekly for 6 weeks and then for 11 weeks improved hyperacusis to be "almost gone." Gopal et al. [51] reported differences in LDLs between conditions in which the patient was unmedicated and medicated with fluvoxamine (50 mg/day) and fluoxetine (20 mg/day), with improved LDLs above $100 \mathrm{~dB}$ in the medicated condition. Lee et al. [53] reported that antihypertensives and gabapentin $(600 \mathrm{mg} /$ day $)$ reduced the intensity of both tinnitus and hyperacusis after 1 month. Nields et al. [50] reported that although six weeks of intravenous ceftriaxone led to a remission of "all symptoms," excluding mild arthralgia and fatigue, hyperacusis subsequently worsened after treatment. Intravenous cefotaxim slightly diminished hyperacusis, but it remained an impediment to performing activities. Clonazepam led to a short-term lessening of hyperacusis symptoms but increased emotional lability. Following carbamazepine (blood level of $6 \mu \mathrm{g} / \mathrm{ml}$.), the patient reported that sound tolerance thresholds had increased, fear and irritability had lessened, and the kindling-like effects (sounds "adding up") had diminished so she was able to recover more quickly.

3.12. Surgery. Six studies reported results from patients electing to undergo surgery specifically for hyperacusis $[56,58,60]$, hearing loss and hyperacusis [57], intracranial aneurysms [55], or Meniere's disease [59] (Table 4). In a cohort study, J. Gavilán and C. Gavilán [59] performed a middle fossa vestibular neurectomy, which includes ablation of the vestibulofacial anastomosis, and removal of part of 
the nerve. They reported that hyperacusis symptoms had lessened or were no longer a problem. Nikkar-Esfahani et al. [56] reported that successful occlusion (permeatal blocking) of the round window surgery in two patients led to an improvement of conductive hyperacusis symptoms but caused a mild exacerbation of conductive hearing loss. Khalil et al. [55] reported a case study of a patient with hyperacusis whose magnetic resonance imaging (MRI) revealed a large aneurysm. A Guglielmi detachable coil (GDC) embolization of the aneurysm led to resolution of hyperacusis. Dang et al. [57] present a case of a patient with hearing loss and hyperacusis caused by bilateral superior semicircular canal dehiscence and posterior semicircular canal dehiscence. Surgery involved a right-sided transmastoid approach and temporalis fascia plugs of each defect. Hyperacusis was assessed as resolved at three months' follow-up. The patient did report residual imbalance and right ear fullness, however. Two of the most recent studies $[58,60]$ reported that transcranial round window niche and oval window reinforcement surgery led to improved hyperacusis, with increased LDLs and reduced Hyperacusis Questionnaire scores (author-developed questionnaire adapted from the GÜF [64]), but with no changes to hearing. One patient reported sustained improvements of hyperacusis and quality of life four years after surgery [58]. Silverstein et al. [60] reported higher success with unilateral surgery than bilateral surgery.

3.13. Future Research Priorities Identified in the Literature. Various further research priorities were identified in the included records. Gabriels [42] urged audiologists to pool their patient information together in order to study the link between symptoms of hyperacusis combined with having a limited DR. Most authors suggest that larger studies (Tables 2-4) need to be conducted to verify the effectiveness of CBT [18], TRT [28, 32], and different surgical treatments [56, 58, $60]$. Studies evaluating the treatment benefit of counselling combined with alternative sound therapies (e.g., enriched acoustic therapy [39]) compared to those usually used in TRT should be conducted [30]. Larger studies focused on testing the effect of maskers/noise generators and HAs specifically for hyperacusis as a primary complaint are indicated [20, 21, 42]. Other possible treatments for hyperacusis suggested for further research include laser therapy [47], desensitisation techniques [41], and further investigation of the pharmaceutical treatments for certain subgroups of hyperacusis specifically when hyperacusis is induced by Lyme disease [50] or when cooccurring with depression [38]. Berry et al. [28] also highlight the importance of developing patient-based assessments for hyperacusis.

\section{Discussion}

This scoping review collated clinical research focused on management strategies used for hyperacusis, the definitions of hyperacusis, tools used for assessment and evaluation, and future research priorities. We found that more than half of the research currently reported was based on individual case studies and therefore cannot be generalised. In addition to this, management strategies were typically evaluated in patients reporting hyperacusis as a secondary complaint or as part of a symptom set, and as such the outcomes reported only provided an indication of effectiveness for hyperacusis. There is a lack of sufficient evidence to identify effective management strategies. These findings highlight an urgent need for controlled trials to evaluate the effectiveness of these management strategies for patients experiencing hyperacusis.

The definitions characterised by researchers had common terminology, with descriptions mainly differing in the emphasis placed on the direction of the sound tolerance, either reduction in tolerance or oversensitivity. The underlying theme reflected how the sound tolerance in patients with hyperacusis is different from "normal." However, the appropriateness of "hypersensitivity" as a term for problems with sound tolerance has been questioned. Tyler et al. [12] argued that hypersensitivity reflects hearing thresholds that are better than normal and that hyperacusis is not usually associated with this and as such recommended avoiding using this terminology. The reference to "abnormal" could be upsetting to patients and lead to negative reactions to the experience. Although some studies referred to emotional reactions, such as distress and discomfort, and physical reactions of pain as characteristics of hyperacusis within their working definitions, the four distinct definitions (loudness, annoyance, fear, and pain) used in a recent narrative review [12] of hyperacusis were not readily used in the literature. The framework suggested by Tyler et al. [12] may be indicative of characteristics of hyperacusis rather than defining discrete subtypes that can be unambiguously differentiated. Consequently, there is a need for consensus through a systematic process involving professionals and patients to define hyperacusis and inform standards for assessment criteria.

To assess hyperacusis, most studies relied on self-report, with only some studies reporting use of a tool (LDLs, the HQ, or the TRT interview) to establish severity. Across the board, no consistent diagnostic tools or criteria were used, making it virtually impossible for comparisons across study populations. There is a need for established diagnostic criteria for hyperacusis and self-report measures appropriate for the population which have been evaluated for this purpose. The same can be said for outcome measures. The majority of studies did not report the outcome measures used. For case reports, the reliance was on clinical interview after treatment, which can be meaningful as the findings directly relate to patient experience. However, with the restricted information reported, we can only have limited confidence in the findings and the possible avenues for future research. Otherwise, a variety of outcome measures were reported, the most popular of which were LDLs and the HQ. Neither has been fully evaluated as outcome measure and in terms of the HQ was not designed to be used as an outcome measure. The three response options only provide coarsegrained categorical units of measurement and as such will not reliably detect small but potentially meaningful changes in hyperacusis $[73,74]$. For LDLs, variability in the type of sounds used and the instructions given to patients' makes comparisons across studies relatively meaningless. There is an apparent need for clear guidance and consensus on LDLs use as outcome measures and for the development of 
self-report questionnaire specifically aimed at being responsive to changes in the impact of hyperacusis.

The most commonly reported management strategy was TRT, and most studies indicated that the treatment was beneficial to patients with hyperacusis. Complex interventions that include several interacting components require systematic evaluations [14]. Most included records reported here lacked rigorous methodology, raising concerns about the validity of the findings. Having said this, the RCTs using patients with a primary complaint [21] or secondary complaint of hyperacusis [30] did provide empirical evidence for the combination of the counselling and sound therapy components (full treatment) being of more benefit than the single components and as such highlighting an important principle of TRT. For the most part, there was a lack of rigor in reporting what was done in the name of TRT, particularly details of the counselling components used throughout the sessions or any differences when treating tinnitus and hyperacusis.

Despite the earliest research reported being published in the 1980s, there is an absence of research evidence on treatments directly aimed at treating hyperacusis. Most studies reported here were not focused on hyperacusis as the primary reason for management. This was especially true for the pharmacological treatments, all of which reported hyperacusis as part of a set of symptoms and were case studies; the effects observed may not be representative of the general hyperacusis population. The drugs reported were in general aimed at alleviating other symptoms and the reduction of hyperacusis may have been a byproduct of the reduction in those symptoms. Without the appropriate population, sample size, and systematic trial methodology, no strong conclusions can be reached.

Overall, only five of the 43 studies used a RCT design $[18,20,21,30,47]$, two of which were solely aimed at patients experiencing hyperacusis $[18,21]$. Whilst there are challenges in utilising a placebo control in interventions that involve sound therapy and/or counselling, waiting list controls designs could be implemented, as could a standard care versus specialist care design. The use of more robust trial designs in future hyperacusis studies would increase the quality and value of the evidence and support the development of optimised treatments.

\section{Conclusions}

Clear themes were identified from the definitions of hyperacusis reported, with an underlying theme that highlights the difference in sound tolerance from what is considered "normal." In order to enable diagnosis and assessment, there needs to be an agreement on the definition including the perspectives of patients with lived experience of hyperacusis. The majority of management strategies were evaluated in patients reporting hyperacusis as a secondary complaint or as part of a symptom set and as such the outcomes only highlight potential benefits and no strong conclusions can be made. Authors agree that research should prioritise evaluating interventions and management strategies on patients with hyperacusis as a primary complaint, in particular TRT, sound therapy interventions, and pharmacological interventions. Importantly, to establish the benefits within this population, there is a need for controlled trials and appropriate patientbased assessments specifically for measuring hyperacusis. To date, only two controlled trials primarily aimed at hyperacusis have been conducted and as yet there are no registered ongoing or planned trials for hyperacusis as a primary complaint. With such an open field, it is essential that these opportunities for new research highlighted here lead to new controlled trials that will have a greater impact on the field.

\section{Disclosure}

The views expressed in this publication are those of the authors and not necessarily those of the NHS, the National Institute for Health Research, the Department of Health.

\section{Conflicts of Interest}

The authors declare that they have no conflicts of interest.

\section{Acknowledgments}

This report is independent research by the National Institute for Health Research Biomedical Research Centre Funding Scheme.

\section{References}

[1] D. M. Baguley and D. J. McFerran, "Hyperacusis and disorders of loudness perception," Textbook of Tinnitus, pp. 13-23, 2011.

[2] D. McFerran, "Misophonia and Phonophobia," in Tinnitus Clin Res Perspect, D. M. Baguley and M. Fagelson, Eds., pp. 245-260, Plural Publishing Inc, San Diego, 2016.

[3] S. Kumar, O. Tansley-Hancock, W. Sedley et al., "The Brain Basis for Misophonia," Current Biology, vol. 27, no. 4, pp. 527-533, 2017.

[4] S. N. Rosing, J. H. Schmidt, N. Wedderkopp, and D. M. Baguley, "Prevalence of tinnitus and hyperacusis in children and adolescents: a systematic review," BMJ Open, vol. 6, no. 6, Article ID e010596, 2016.

[5] A. J. Hall, R. Humphriss, D. M. Baguley, M. Parker, and C. D. Steer, "Prevalence and risk factors for reduced sound tolerance (hyperacusis) in children," International Journal of Audiology, vol. 55, no. 3, pp. 135-141, 2016.

[6] G. Andersson, N. Lindvall, T. Hursti, and P. Carlbring, "Hypersensitivity to sound (hyperacusis): a prevalence study conducted via the internet and post," International Journal of Audiology, vol. 41, no. 8, pp. 545-554, 2002.

[7] J. Paulin, L. Andersson, and S. Nordin, "Characteristics of hyperacusis in the general population," Noise and Health, vol. 18, no. 83, pp. 178-184, 2016.

[8] M. Anari, A. Axelsson, A. Eliasson, and L. Magnusson, "Hypersensitivity to sound-questionnaire data, audiometry and classification," Scandinavian Audiology, vol. 28, no. 4, pp. 219-230, 1999.

[9] P. J. Jastreboff and M. M. Jastreboff, "Tinnitus retraining therapy (TRT) as a method for treatment of tinnitus and hyperacusis patients," Journal of the American Academy of Audiology, vol. 11, no. 3, pp. 162-177, 2000. 
[10] J. Sheldrake, P. U. Diehl, and R. Schaette, "Audiometric characteristics of hyperacusis patients," Frontiers in Neurology, vol. 6, Article ID 00105, 2015.

[11] M. Knipper, P. Van Dijk, I. Nunes, L. Rüttiger, and U. Zimmermann, "Advances in the neurobiology of hearing disorders: recent developments regarding the basis of tinnitus and hyperacusis," Progress in Neurobiology, vol. 111, pp. 17-33, 2013.

[12] R. S. Tyler, M. Pienkowski, E. R. Roncancio et al., "A review of hyperacusis and future directions: part I. Definitions and manifestations," American Journal of Audiology, vol. 23, no. 4, pp. 402-419, 2014.

[13] H. Aazh, D. McFerran, R. Salvi, D. Prasher, M. Jastreboff, and P. Jastreboff, "Insights from the first international conference on hyperacusis: causes, evaluation, diagnosis and treatment," Noise and Health, vol. 16, no. 69, pp. 123-126, 2014.

[14] P. Craig, P. Dieppe, S. Macintyre, S. Mitchie, I. Nazareth, and M. Petticrew, "Developing and evaluating complex interventions: the new Medical Research Council guidance," British Medical Journal, vol. 337, Article ID a1655, 2008.

[15] H. Arksey and L. O’Malley, "Scoping studies: towards a methodological framework," International Journal of Social Research Methodology, vol. 8, no. 1, pp. 19-32, 2005.

[16] M. D. J. Peters, C. M. Godfrey, H. Khalil, P. McInerney, D. Parker, and C. B. Soares, "Guidance for conducting systematic scoping reviews," International Journal of Evidence-Based Healthcare, vol. 13, no. 3, pp. 141-146, 2015.

[17] E. Aromataris and D. Riitano, "Constructing a search strategy and searching for evidence," American Journal of Nursing, vol. 114, no. 5, pp. 49-56, 2014.

[18] L. Jüris, G. Andersson, H. C. Larsen, and L. Ekselius, "Cognitive behaviour therapy for hyperacusis: a randomized controlled trial," Behaviour Research and Therapy, vol. 54, no. 1, pp. 30-37, 2014.

[19] A. B. Fioretti, T. Varakliotis, O. Poli, M. Cantagallo, and A. Eibenstein, "Severe hyperacusis, photophobia, and skin hypersensitivity," Case Reports in Otolaryngology, vol. 2016, pp. 1-5, 2016.

[20] W. Hiller and C. Haerkötter, "Does sound stimulation have additive effects on cognitive-behavioral treatment of chronic tinnitus?" Behaviour Research and Therapy, vol. 43, no. 5, pp. 595-612, 2005.

[21] C. Formby, M. Hawley, L. Sherlock et al., "Intervention for restricted dynamic range and reduced sound tolerance," The Journal of the Acoustical Society of America, vol. 123, no. 5, pp. 3717-3717, 2008.

[22] S. Gold, E. Frederick, and C. Formby, "Shifts in dynamic range for hyperacusis patients reveiving tinnitus retraining therapy (TRT)," in Sixth Int Tinnitus Semin, J. Hazell, Ed., pp. 297-301, Cambridge, UK, 2002.

[23] C. Wölk and B. Seefeld, "The effects of managing hyperacusis with maskers (noise generators)," in Proc Sixth Int Tinnitus Semin, J. Hazell, Ed., pp. 512-514, Cambridge, UK, 2002.

[24] J. Hazell, J. Sheldrake, and R. Graham, "Decreased sound tolerance: predisposing factors, triggers and outcomes after TRT,' in Seventh Int Tinntius Semin, pp. 255-261, 2002.

[25] P. J. Jastreboff and M. M. Jastreboff, "Treatments for decreased sound tolerance (hyperacusis and misophonia)," Seminars in Hearing, vol. 35, no. 2, pp. 105-120, 2014.

[26] S. Forti, S. Costanzo, A. Crocetti, L. Pignataro, L. Del Bo, and U. Ambrosetti, "Are results of tinnitus retraining therapy maintained over time? 18-Month follow-up after completion of therapy," Audiology and Neurotology, vol. 14, no. 5, pp. 286-289, 2009.

[27] E. Molini, M. Faralli, C. Calenti, G. Ricci, F. Longari, and A. Frenguelli, "Personal experience with tinnitus retraining therapy," European Archives of Oto-Rhino-Laryngology, vol. 267, no. 1, pp. 51-56, 2010.

[28] J. A. Berry, S. L. Gold, E. A. Frederick, W. C. Gray, and H. Staecker, "Patient-based outcomes in patients with primary tinnitus undergoing tinnitus retraining therapy," Archives of Otolaryngology-Head \& Neck Surgery, vol. 128, no. 10, pp. 11531157, 2002.

[29] L. Suchova, "Tinnitus retraining therapy-the experiences in Slovakia.," Bratislavske lekarske listy, vol. 106, no. 2, pp. 79-82, 2005.

[30] C. Formby, M. L. Hawley, L. P. Sherlock et al., "A sound therapybased intervention to expand the auditory dynamic range for loudness among persons with sensorineural hearing losses: A randomized placebo-controlled clinical trial," Seminars in Hearing, vol. 36, no. 2, pp. 77-110, 2015.

[31] C. McKinney, J. Hazell, and R. Graham, "Changes in loudness discomfprt level and sensitivity to environmental sound," in Proc Sixth Int Tinnitus Semin, J. Hazell, Ed., pp. 499-501, Cambridge, UK, 2002.

[32] G. Bartnik, A. Fabijańska, and M. Rogowski, "Experiences in the treatment of patients with tinnitus and/or hyperacusis using the habituation method," Scandinavian Audiology, Supplement, vol. 30, no. 52, pp. 187-190, 2001.

[33] C. Formby and M. L. Keaser, "Secondary treatment benefits achieved by hearing-impaired tinnitus patients using aided environmental sound therapy for tinnitus retraining therapy: Comparisons with matched groups of tinnitus patients using noise generators for sound therapy," Seminars in Hearing, vol. 28, no. 4, pp. 246-294, 2007.

[34] C. Formby and S. L. Gold, "Modification of loudness discomfort level: Evidence for adaptive chronic auditory gain and its clinical relevance," Seminars in Hearing, vol. 23, no. 1, pp. 21-34, 2002.

[35] G. Hesse, A. Laubert, H. Schaaf, and M. Almeling, "Integrative psychosomatic and otologic models in the therapy of chronic tinnitus," in Trends Psychother Res Nova Science Publishers Inc, M. E. Abelian, Ed., pp. 53-70, 2006.

[36] M. Westcott, Case study: management of hyperacusis associated with post-traumatic stress disorder, Seventh Int Tinntius Semin, 2002.

[37] R. A. Ruth and R. Hamill-Ruth, "A multidisciplinary approach to management of Tinnitus and hyperacusis," Hearing Journal, vol. 54, no. 11, pp. 26-32, 2001.

[38] D. Attri and A. N. Nagarkar, "Resolution of hyperacusis associated with depression, following lithium administration and directive counselling," Journal of Laryngology and Otology, vol. 124, no. 8, pp. 919-921, 2010.

[39] A. J. Noreña and S. Chery-Croze, "Enriched acoustic environment rescales auditory sensitivity," NeuroReport, vol. 18, no. 12, pp. 1251-1255, 2007.

[40] C. Miani, P. Passon, A. M. Bergamin Bracale, A. Barotti, and N. Panzolli, "Treatment of hyperacusis in Williams syndrome with bilateral conductive hearing loss," European Archives of OtoRhino-Laryngology, vol. 258, no. 7, pp. 341-344, 2001.

[41] J. A. Vernon, "Pathophysiology of tinnitus: a special case-hyperacusis and a proposed treatment," American Journal of Otology, vol. 8, no. 3, pp. 201-202, 1987.

[42] P. Gabriels, "Hyperacusis - can we help?" Aust J Audiol, vol. 15, no. 1, pp. 1-4, 1993. 
[43] J. Vernon and L. Press, “Treatment for hyperacusis," in Tinnitus: Treatment and Relief, J. Vernon, Ed., pp. 223-227, Allyn and Bacon, Boston, 1998.

[44] A. Ramos Macías, J. C. Falcón González, M. Manrique et al., "Cochlear implants as a treatment option for unilateral hearing loss, severe tinnitus and hyperacusis," Audiology and Neurotology, vol. 20, pp. 60-66, 2015.

[45] G. Mertens, M. De Bodt, and P. Van de Heyning, "Cochlear implantation as a long-term treatment for ipsilateral incapacitating tinnitus in subjects with unilateral hearing loss up to 10 years," Hearing Research, vol. 331, pp. 1-6, 2016.

[46] C. Saglier, F. Perez-Diaz, L. Collet, and R. Jouvent, "The impact of a family history hearing impairement on rehabilative intervention: a one year follow up," in Eff Genet Heaing Impair Fam, D. Stephens and L. Jones, Eds., pp. 135-144, John Wiley and Sons, Chichester, UK, 2006.

[47] R. Teggi, C. Bellini, L. O. Piccioni, F. Palonta, and M. Bussi, "Transmeatal low-level laser therapy for chronic tinnitus with cochlear dysfunction," Audiology and Neurotology, vol. 14, no. 2, pp. 115-120, 2009.

[48] O. Meeus, K. Heyndrickx, P. Lambrechts, D. De Ridder, and P. Van De Heyning, "Phase-shift treatment for tinnitus of cochlear origin," European Archives of Oto-Rhino-Laryngology, vol. 267, no. 6, pp. 881-888, 2010.

[49] M. Valente, J. Goebel, D. Duddy, B. Sinks, and J. Peterein, "Evaluation and treatment of severe hyperacusis," J Am Acad Audiol, vol. 11, no. 6, pp. 295-299, 2000.

[50] J. A. Nields, B. A. Fallon, and P. J. Jastreboff, "Carbamazepine in the treatment of Lyme disease-induced hyperacusis," Journal of Neuropsychiatry and Clinical Neurosciences, vol. 11, no. 1, pp. 97-99, 1999.

[51] K. Gopal, D. M. Daly, R. G. Daniloff, and L. Pennartz, "Effects of selective serotonin reuptake inhibitors on auditory processing," The Journal of the Acoustical Society of America, vol. 105, no. 2, pp. 1346-1347, 1999.

[52] K. H. Brookler, "Electronystagmography in a woman with aural fullness, hyperacusis, and dizziness," Ear, Nose and Throat Journal, vol. 82, no. 3, pp. 165-166, 2003.

[53] E. Lee, H.-Y. Sohn, M. Kwon, and J. S. Kim, "Contralateral hyperacusis in unilateral pontine hemorrhage.," Neurology, vol. 70, no. 24, pp. 2413-2415, 2008.

[54] K. H. Brookler, "Monaural diplacusis with tinnitus, aural fullness, hyperacusis, and sensorineural hearing loss," Ear, Nose and Throat Journal, vol. 88, no. 2, pp. 772-774, 2009.

[55] S. Khalil, L. Ogunyemi, and J. Osborne, "Middle cerebral artery aneurysm presenting as isolated hyperacusis," Journal of Laryngology and Otology, vol. 116, no. 5, pp. 376-378, 2002.

[56] A. Nikkar-Esfahani, D. Whelan, and A. Banerjee, "Occlusion of the round window: A novel way to treat hyperacusis symptoms in superior semicircular canal dehiscence syndrome," Journal of Laryngology and Otology, vol. 127, no. 7, pp. 705-707, 2013.

[57] P. T. Dang, T. A. Kennedy, and S. P. Gubbels, "Simultaneous, unilateral plugging of superior and posterior semicircular canal dehiscences to treat debilitating hyperacusis," Journal of Laryngology and Otology, vol. 128, no. 2, pp. 174-178, 2014.

[58] H. Silverstein, Y.-H. E. Wu, and S. Hagan, "Round and oval window reinforcement for the treatment of hyperacusis," American Journal of Otolaryngology - Head and Neck Medicine and Surgery, vol. 36, no. 2, pp. 158-162, 2015.

[59] J. Gavilán and C. Gavilán, "Middle fossa vestibular neurectomy: long-term results," Archives of Otolaryngology, vol. 110, no. 12, pp. 785-787, 1984.
[60] H. Silverstein, R. Ojo, J. Daugherty, R. Nazarian, and J. Wazen, "Minimally invasive surgery for the treatment of hyperacusis," Otology and Neurotology, vol. 37, no. 10, pp. 1482-1488, 2016.

[61] P. Jastreboff, "Clinical implication of the neurophysiological model of tinnitus," in Proc 5th Int Tinnitus Semin Portland, G. Reich and J. Vernon, Eds., pp. 500-507, 1995.

[62] M. Nelting, N. K. Rienhoff, G. Hesse, and U. Lamparter, "The assessment of subjective distress related to hyperacusis with a self-rating questionnaire on hypersensitivity to sound," Laryngo-Rhino- Otologie, vol. 81, no. 5, pp. 327-334, 2002.

[63] S. Khalfa, S. Dubal, E. Veuillet, F. Perez-Diaz, R. Jouvent, and L. Collet, "Psychometric normalization of a hyperacusis questionnaire," ORL, vol. 64, no. 6, pp. 436-442, 2002.

[64] M. Nelting and N. Finlayson, GÜF-Geräuschüberempfindlichkeits-Fragebogen, Göttingen, Hogrefe, Germany, 2004.

[65] P. J. Jastreboff and J. W. Hazell, Tinnitus Retraining Therapy: Implementing the Neurophysiological Model, Cambridge University Press, Cambridge, UK, 2004.

[66] W. Hiller and G. Goebel, "A psychometric study of complaints in chronic tinnitus," Journal of Psychosomatic Research, vol. 36, no. 4, pp. 337-348, 1992.

[67] R. Dauman and F. Bouscau-Faure, "Assessment and amelioration of hyperacusis in tinnitus patients," Acta OtoLaryngologica, vol. 125, no. 5, pp. 503-509, 2005.

[68] K. Fackrell, C. Fearnley, D. J. Hoare, and M. Sereda, "Hyperacusis questionnaire as a tool for measuring hypersensitivity to sound in a tinnitus research population," BioMed Research International, vol. 2015, Article ID 290425, 12 pages, 2015.

[69] B. Goldstein and A. Shulman, "Tinnitus-hyperacusis and the loudness discomfort level test-a preliminary report," Int Tinnitus J, vol. 2, pp. 83-89, 1996.

[70] L. P. Sherlock and C. Formby, "Estimates of loudness, loudness discomfort, and the auditory dynamic range: Normative estimates, comparison of procedures, and test-retest reliability," Journal of the American Academy of Audiology, vol. 16, no. 2, pp. 85-100, 2005.

[71] S. P. Bornstcin and F. E. Musick, "Loudness Discomfort Level and Reliability As A Function of Instructional Set," Scandinavian Audiology, vol. 22, no. 2, pp. 125-131, 1993.

[72] T. L. Zaugg, E. J. Thielman, S. Griest, and J. A. Henry, "Subjective reports of trouble tolerating sound in daily life versus loudness discomfort levels," American Journal of Audiology, vol. 25, no. 4, pp. 359-363, 2016.

[73] M. W. Lipsey and S. M. Hurley, "Design sensitivity: statistical power for applied experimental research," SAGE Handb Appl Soc Res methods, pp. 44-76, 2008.

[74] B. Kirshner and G. Guyatt, "A methodological framework for assessing health indices," Journal of Chronic Diseases, vol. 38, no. 1, pp. 27-36, 1985. 


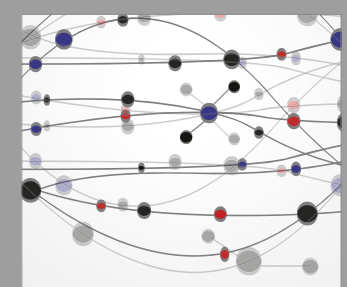

The Scientific World Journal
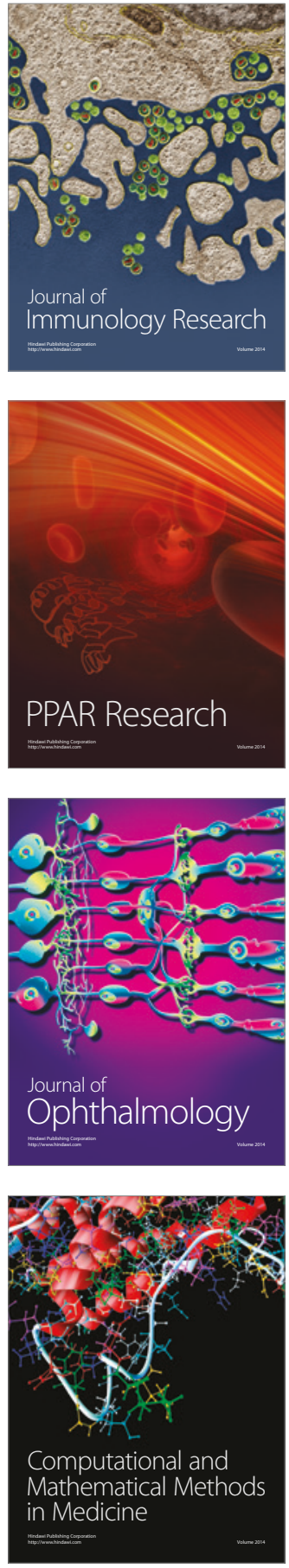

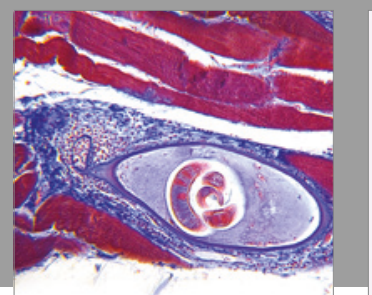

Gastroenterology Research and Practice
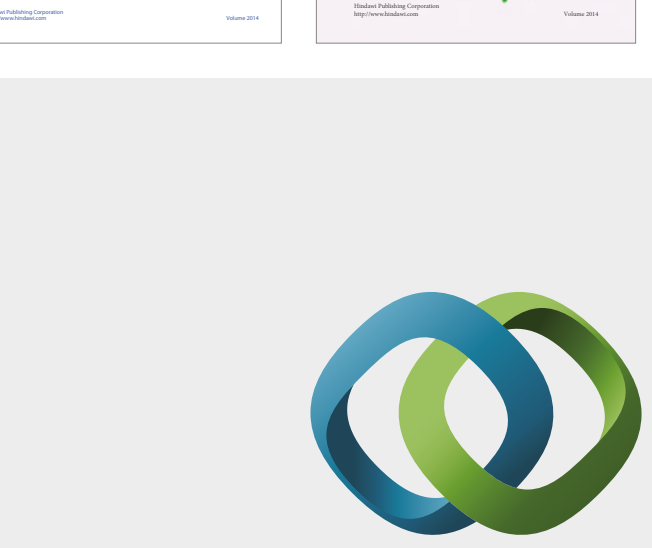

\section{Hindawi}

Submit your manuscripts at

https://www.hindawi.com
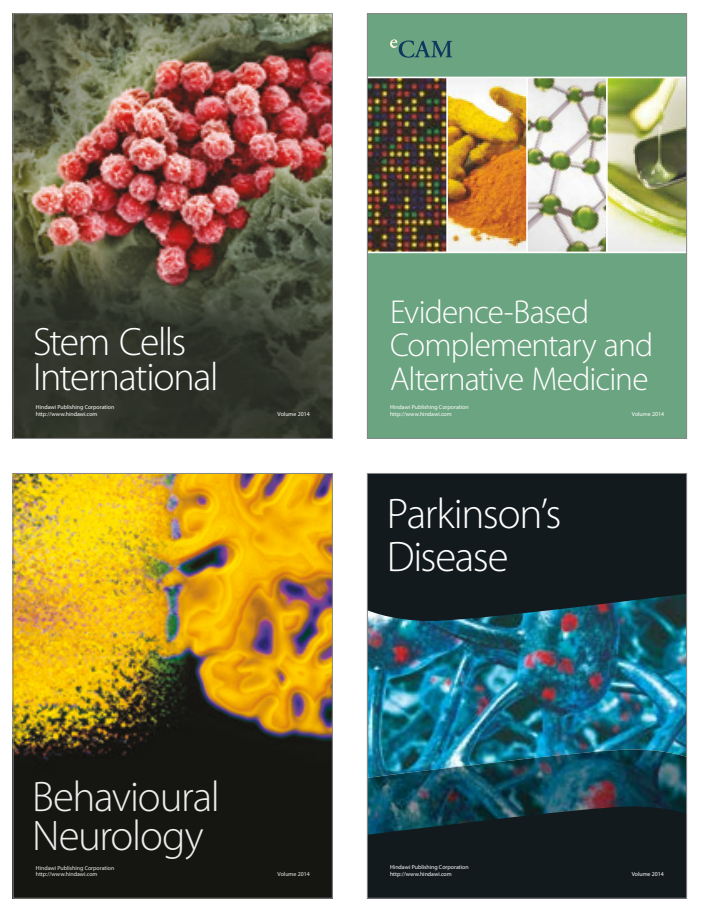
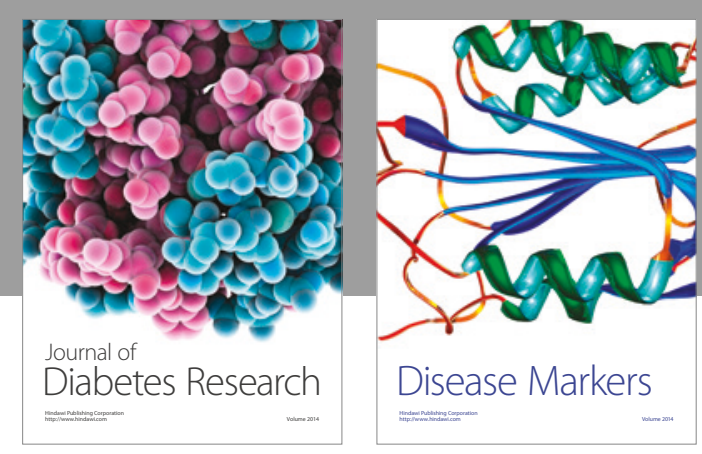

Disease Markers
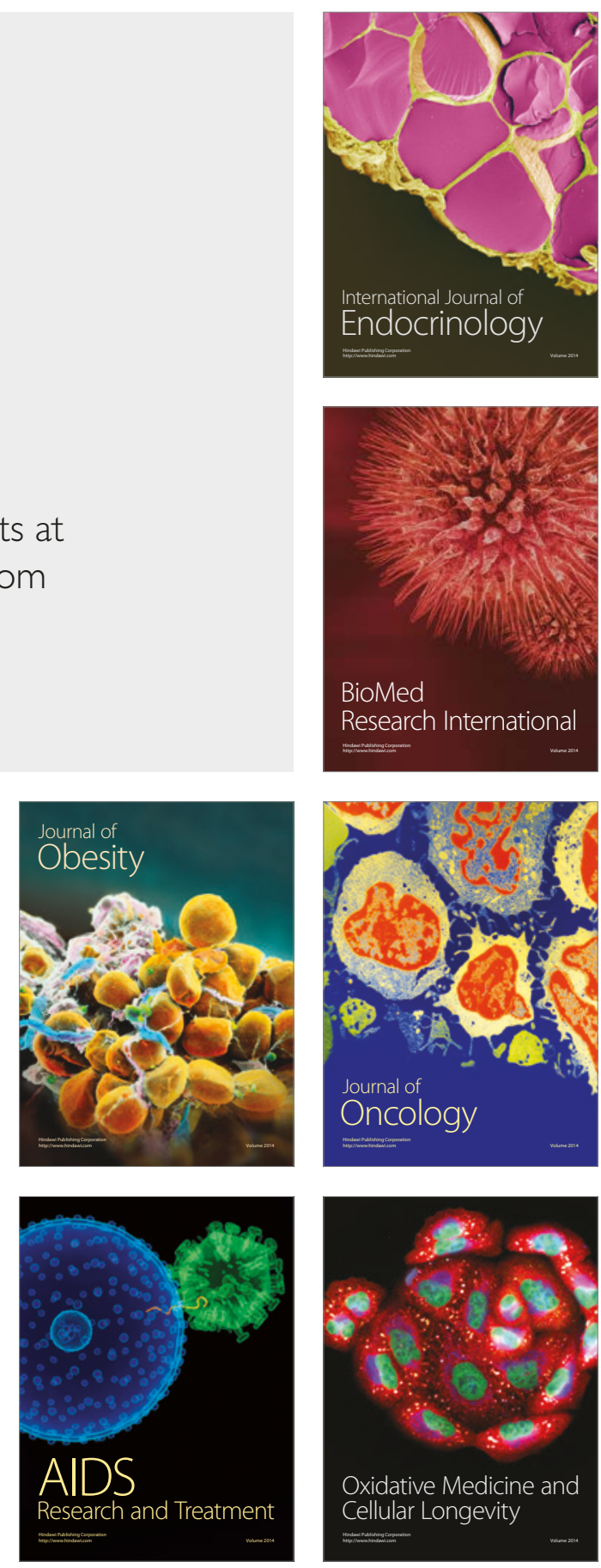